\title{
Experimental study of the turbulence intensity effects on marine current turbines behaviour. Part II: Two interacting turbines
}

\author{
Paul Mycek $^{\mathrm{a}, \mathrm{b}}$, Benoît Gaurier ${ }^{\mathrm{b}}$, Grégory Germain $^{\mathrm{b}}$, Grégory Pinon $^{\mathrm{a}, *}$, Elie Rivoalen ${ }^{\mathrm{a}, \mathrm{c}}$ \\ a Laboratoire Ondes et Milieux Complexes, UMR 6294, CNRS e Université du Havre, 53, rue de Prony, BP 540, \\ F-76058 Le Havre Cedex, France \\ ${ }^{b}$ IFREMER, Marine Structures Laboratory, 150, quai Gambetta, BP 699, F-62321 Boulogne-Sur-Mer, France \\ ${ }^{\mathrm{c}}$ Laboratoire d'Optimisation et Fiabilité en Mécanique des Structures, EA 3828, INSA de Rouen, Avenue de \\ I'Université, BP 08, F-76801 Saint-Etienne-du-Rouvray, France \\ *: Corresponding author : Grégory Pinon, email address : gregory.pinon@univ-lehavre.fr \\ paul.mycek@ifremer.fr ; benoit.gaurier@ifremer.fr ; gregory.germain@ifremer.fr ; elie.rivoalen@insa-rouen.fr
}

\begin{abstract}
:
The future implantation of second generation marine current turbine arrays depends on the understanding of the negative interaction effects that exist between turbines in close proximity. This is especially the case when the turbines are axially aligned one behind another in the flow.

In order to highlight these interaction effects, experiments were performed in a flume tank on 3-bladed $1 / 30$ th scale prototypes of horizontal axis turbines.

This work focuses on the interactions between two horizontal axis marine current turbines, axially aligned with the upstream flow. Thrust and power coefficients function of the rotation speed of the downstream device are presented. Besides, the wake of each turbine is characterised so as to explain their behaviour.

A large range of inter-device distances is considered, as well as two upstream turbulence intensity conditions, namely $3 \%$ and $15 \%$. This latter parameter deeply influences the behaviour of a marine current turbine and thus plays a preponderant role in the interactions effects between two devices. Indeed, this study points out that, for the considered turbine and blade geometry, higher ambient turbulence intensity rates $(15 \%)$ reduce the wake effects, and thus allows a better compromise between inter-device spacing and individual performance.
\end{abstract}

\section{Highlights}

- Interaction effects between two aligned 3-bladed horizontal axis marine current turbines are considered. - Two ambient turbulence intensity rates are considered. The wake and performances of the turbine are characterised. A wide range of inter-device distances is considered. The ambient turbulence intensity deeply influences the interaction effects.

Keywords : Marine current turbine ; Performance ; Wake ; Turbulence ; Array ;Tidal turbine

\section{Introduction}

The ambient turbulence intensity in the upstream flow plays a decisive role in the behaviour of horizontal axis marine current turbines. First, turbulence intensity influences the turbine performance but, probably most important, it deeply influences the wake shape [1] and [2]. This last issue is of 
crucial matter for the onset of marine current turbine arrays. Indeed, in second generation arrays, the wake of an upstream turbine may irreparably affect the power performance of another turbine positioned downstream. The present paper aims at characterising precisely the performance and the wake of two interacting turbines aligned with the flow direction. This configuration represents the easiest, but strongest negative interaction possible between two turbines. The first part of this study [1] was dedicated to a precise characterisation of the performance and wake of a single turbine depending on the ambient turbulence intensity of the incoming flow. These previous results would represent a strong basis for comparisons with the twin turbines setups investigated here.

Concerning turbines array configurations, some general guidelines and general considerations have been proposed for the performance assessment in such arrays [3-5]. However, real experimental trials on marine current turbines in an array are still rather seldom [6-8]. In fact, real turbine interactions within a flume tank require large experimental devices and facilities, which are not widely spread and almost impossible to carry out in a towing tank. The first study by Jo et al. [6] is very similar to ours regarding the turbine configurations. However, their paper is not detailed enough, which prevents us from making comparisons with our results. In addition, most of their results were extrapolated data from numerical computations using ANSYS CFX. Stallard et $a l$. $[7,8]$ carried out a precise experimental study of several 3-bladed turbines immersed in a flume tank. They documented wake velocity profiles, including turbulent intensities in the wake of turbine arrays. Although they mention several configurations of arrays, including two-rowed arrays, the presented results deal with velocity and turbulence intensity profiles of single-rowed turbine arrays with different lateral distances. To our knowledge, there is no detailed study dealing with such elementary interactions between axially aligned turbines, considering both performance and wake analysis.

Probably owing to the reasons discussed above (size of the experimental device, cost of such structures, etc.) most papers dealing with marine current turbine arrays are numerical studies. Malki et al. [9] performed some 3D BEM-CFD (Blade Element Momentum theory - Computational Fluid Dynamics) computations for several configurations of a 3-turbine array, the third turbine being in a staggered position in the second row. They discuss the performances and the velocity deficit of such arrays, mainly depending on the first row lateral spacing between turbines. O'Doherty et al. [10] presented a similar study with a full 3D CFD solver (including rotating blades) for two configurations of 4-turbine arrays; the first 3 turbines making up the first row, the fourth one being either staggered or aligned in the second row. They also discuss performance and wake analysis in an interesting manner. More recently, Churchfield et al. [11] performed impressive computations of up to five turbines in a two-rowed array. Their 3D solver takes a special care of turbulence modelisation (Large Eddy Simulation model) and the 2-bladed turbines are taken into account with a kind of Blade Element Momentum theory. They show vertical and horizontal velocity profiles together with an analysis of the array global performance. In their conclusion, these researchers clearly ask for experimental data of interacting turbines in order to compare their results with. The presented experimental results will hopefully give them some material. Divett et al. [12] also investigated numerical computation of multiple turbine arrays, consisting of up to 15 turbines. Their computations were obtained with the Gerris software (an adaptive mesh solver), under the porous disc approximation as Harrison et al. did in [13], and they mainly discuss about the array global performance. Trowse and Krasten [14] carried out a numerical study on maximum extractable power from 300 turbines in the Minas Passage (Bay of Fundy, Canada) together with studies on environmental and socio-economic impacts. They used a meso-scale software to compute the flow modifications due to the presence of these turbines in the Minas Passage. In a recent paper, Karsten 
et al. [15] present numerical computations with up to a thousand turbines in the Minas Passage. Roc et al. [16] recently discussed the tidal turbine representation in such software. The results of the present study will, in our opinion, provide interesting data sets for further validation of turbine interactions with numerical tools.

The present study aims at characterising the interaction effects between two horizontal axis turbines, in terms of power and thrust coefficients $\left(C_{P}\right.$ and $\left.C_{T}\right)$ modification together with detailed wake profiles, including turbulence intensities. This paper follows the same experimental procedures as presented in $[1,2]$, but with an open-modified version of the turbine which enables the diffusion of the blades geometry. Some of the experimental results were partially presented or used as a matter of numerical-experimental validation in $[17,18]$. The present document presents all our latest experimental results of two 3-bladed turbines aligned with the flow and immersed in two different turbulence intensities, namely $I_{\infty}=3 \%$ and $I_{\infty}=15 \%$.

First of all, section 2 details the experimental setup, the measurement techniques as well as the turbines configurations and geometry. Section 3 presents the power and thrust coefficients evolutions, for different incoming mean velocities, different inter-device spacings and the two turbulence intensity rates. The standard deviations of these coefficients are also plotted. Then, section 4 gives streamwise velocity, turbulence intensity and Reynolds shear stress maps depending on the two turbulence intensity rates. The wake is also characterised using integrated quantities. Finally, most of the raw results are made available in the appendices as a matter of validation with future numerical studies. This intends to answer to recurrent requests, the latest being by Churchfield et al. [11].

\section{Experiments description}

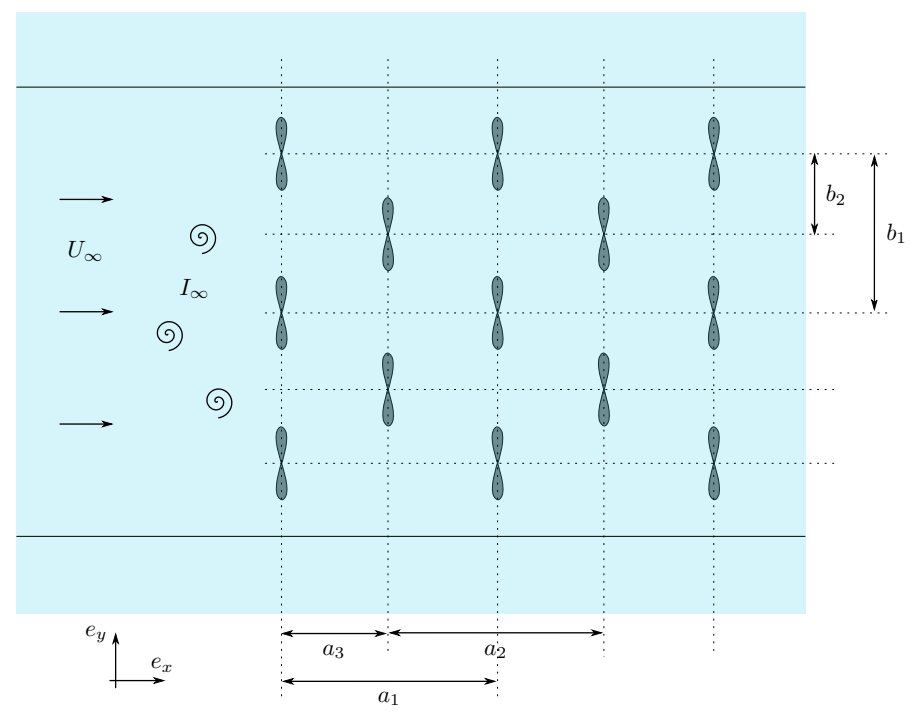

Figure 1: Schematic top view of a marine current turbine array.

The present study deals with the characterisation of the interaction effects between marine 
current turbines. Figure 1 depicts a general scheme of turbines array, as every one can find in the literature [3-5]. Here, a special focus is made on elementary interactions between two marine current turbines placed one behind another and axially aligned with the flow. In that case, since parameter $a_{1}$ alone can describe the configuration, the inter-device spacing will simply be referred to as $a$.

\subsection{Experimental setup}

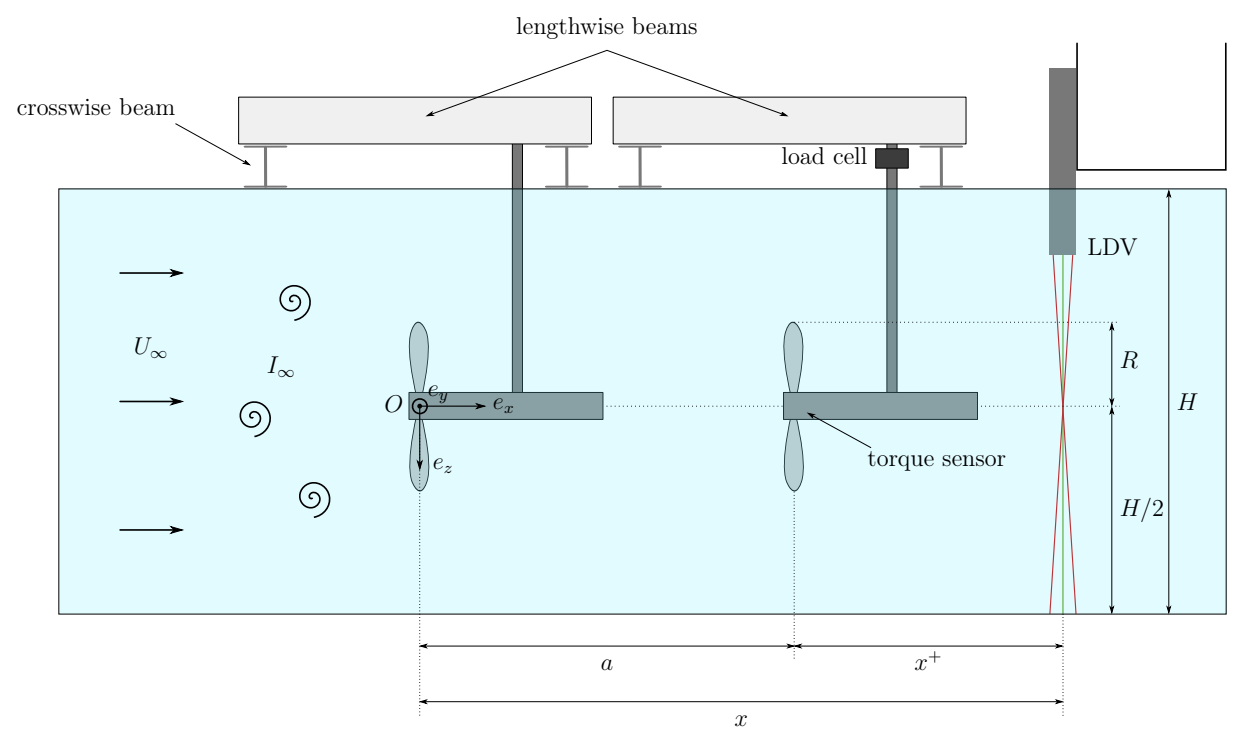

Figure 2: Schematic view of the twin turbines setup with $a>5 D$. For $a \leq 5 D$, the two lengthwise I-beams are replaced by a single longer lengthwise I-beam, on which both turbines are fixed (cf. Figure 3). The origin $O(0,0,0)$ is chosen at the upstream turbine rotor centre.

The two turbines used for this work are identical, in terms of geometry (blades and hub), to the one defined in the first part of this study, Part I: one Single Turbine [1]. The upstream flow conditions are those presented in the previous paper as well, except that only one upstream velocity is considered, namely $U_{\infty}=0.8 \mathrm{~m} \cdot \mathrm{s}^{-1}$ for $I_{\infty}=3 \%$ and $U_{\infty}=0.83 \mathrm{~m} \cdot \mathrm{s}^{-1}$ for $I_{\infty}=15 \%$.

Each turbine model is fixed to a lengthwise I-beam by means of a mast. Both masts are of equal length. The I-beams are placed over the flume tank, parallel to the upstream current and at equal distance from both sides, as shows the schematic side-view depicted on Figure 2. When the inter-device distance equals $5 D$ or lower, the two lengthwise I-beams are replaced by a single longer lengthwise I-beam, as shows the picture depicted on Figure 3, for $a=4 D$. In fact, Figure 3 shows a picture of both turbines out of the water.

The origin $O(0,0,0)$ is chosen at the upstream rotor centre, such that the axial distance from the upstream turbine is denoted by $x$, the downstream turbine is placed at $x=a$, and thus the axial distance from downstream turbine, denoted by $x^{+}$is such that $x=a+x^{+}$. Lengths being still made dimension-free by the rotor diameter $D$, let $a^{*}=a / D$ and $x^{+*}=x^{+} / D$.

One may refer to Part I [1] of the present study for further details about the flume tank, turbine models, measurement techniques and material, as well as the upstream and downstream flow characteristics. 


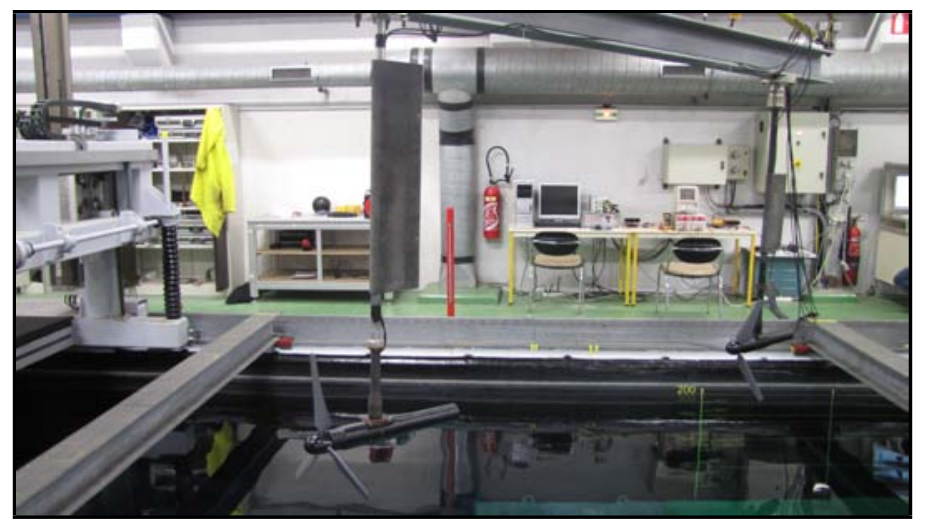

Figure 3: Photography of the twin turbines setup with $a=4 D$. Both turbines are fixed on the same lengthwise I-beam.

\subsection{Force and moment measurements}

During these tests, only the second turbine is instrumented in order to quantify the interaction effects in terms of $C_{P}$ and $C_{T}$ with the use of a torque meter and a six-component load cell, as described in [1]. The measurement (and thus the averaging) duration is $T=100$ seconds.

The definition of downstream indicators such as the TSR or the thrust and power coefficients is a delicate matter. On the one hand, these coefficients are supposed to characterise a turbine independently of the flow conditions (provided that they belong to the operating range). These coefficients should thus be defined in relation to the flow conditions that are perceived by the downstream turbine. By doing so, the evolution of the thrust and power coefficients function of the TSR should all remain similar to the one of a single turbine. The potential discrepancies may stem from two reasons: either the perceived velocity is out of the operating range of the turbine, or the perceived flow is so perturbed (heterogeneous flow, presence of coherent structures) that the velocity alone does not suffice to characterise the turbine behaviour anymore. This first approach allows to study the individual marine current turbine behaviour in an interaction (array) configuration.

On the other hand, the flow conditions may not be deducted or predicted easily at every potential location for a downstream turbine. It is then interesting to consider the above mentioned coefficients defined in relation to the flow conditions upstream of the whole farm (that is, in our case, before the upstream turbine). Their evolution should thus be extremely different from each other since they are not based on the flow the turbine actually perceives. However, this approach may provide valuable information on the overall array efficiency, with only the knowledge of the farm upstream flow conditions being required.

In the present study, both approaches will be considered, which is why generic downstream coefficients are defined, depending on the flow condition (more precisely the flow velocity $u$ ) that is used for the computation. First, the downstream TSR, based on the velocity value $u$, is defined by:

$$
\mathrm{TSR}_{u}^{\text {down }}=\frac{\left|\Omega_{x}^{\text {down }}\right| R}{u}
$$

where $\Omega_{x}^{\text {down }}$ denotes the axial rotation speed of the rotor. Likewise, the downstream power coeffi- 
cient based on $u$ is given by:

$$
C_{P, u}^{\text {down }}=\frac{\mathcal{P}^{\text {down }}}{\mathcal{P}_{u}}=\frac{\mathcal{M}_{x}^{\text {down }} \Omega_{x}^{\text {down }}}{\frac{1}{2} \rho \pi R^{2} u^{3}},
$$

where $\mathcal{P}^{\text {down }}$ denotes the power retrieved by the downstream turbine and $\mathcal{M}_{x}^{\text {down }}$ denotes its axial moment. $\mathcal{P}_{u}$ represents the maximum power available from a flow with an homogeneous streamwise velocity $u$. Finally, the downstream thrust coefficient based on $u$ is defined as follows:

$$
C_{T, u}^{\text {down }}=\frac{\mathcal{F}_{x}^{\text {down }}}{\frac{1}{2} \rho \pi R^{2} u^{2}},
$$

where $\mathcal{F}_{x}^{\text {down }}$ denotes the axial force on the downstream device. The $\mathcal{F}_{x}^{\text {down }}$ measured here actually includes the axial force on the whole structure, that is the blades, the hub and the mast.

Natural choices of $u$ are thus either $U_{\infty}$ or $\bar{u}_{0}$ or $\hat{\bar{u}}_{r}$, with $r=R$ or $R^{+}$following the notations given in Part I [1] and redefined in section 2.3. For consistence and clarity, the TSR of the upstream turbine will be denoted by $\mathrm{TSR}^{u p}$ from now on. Likewise, the power coefficient and the thrust coefficient of a single turbine (or equivalently of the unperturbed upstream turbine) will be denoted by $C_{P}^{\text {single }}$ and $C_{T}^{\text {single }}$ respectively.

\section{3. $L D V$ measurements}

The flow velocity measurements are performed by means of a Laser Doppler Velocimetry (LDV) system described in $[2,19]$. The laser used for the LDV was presented in [1]. The LDV measurements are performed on a grid whose nodes $\left(X_{i}, Y_{i}\right)$ are arranged as follows:

- $X_{1}=1.2 D$ and $X_{i}=i \times D$ for $i=2, \ldots, 10$;

- $Y_{i}=-1.2+(i-1) \times 0.1 \mathrm{~m}$ for $i=1, \ldots, 25$, with two additional positions $Y_{26}=-Y_{27}=R=$ $0.35 \mathrm{~m}$.

The measurement duration on each node is $T=100$ seconds with an observed data rate between 5 and $54 \mathrm{~Hz}$.

Some important definitions from Part I [1] are briefly recalled. The centreline axial velocity at a given location $x$ downstream of the turbine is defined by:

$$
\bar{u}_{0}(x)=\bar{u}(x, 0,0) .
$$

The corresponding centreline axial velocity deficit, expressed as a percentage, is basically given by:

$$
\gamma_{0}(x)=100\left(1-\frac{\bar{u}_{0}(x)}{U_{\infty}}\right) .
$$

Likewise, the centreline turbulence intensity rate is defined as:

$$
I_{0}(x)=I(x, 0,0) .
$$

Now, corresponding disc-integrated values are defined:

$$
\hat{\bar{u}}_{r}^{*}(x)=\frac{\hat{\bar{u}}_{r}(x)}{U_{\infty}} \simeq \frac{1}{r^{2} U_{\infty}} \int_{-r}^{r}|y| \bar{u}(x, y, 0) d y,
$$


where $\hat{\bar{u}}_{r}(x) \simeq \frac{1}{r^{2}} \int_{-r}^{r}|y| \bar{u}(x, y, 0) d y$ represents a normalised, dimensional disc-integrated velocity such that $\hat{\bar{u}}_{r}(x)=U_{\infty}$ when $\bar{u}(x, y, 0)=U_{\infty}$ (whatever $\left.y \in[-r ; r]\right)$. The disc-integrated axial velocity deficit is the given by:

$$
\hat{\gamma}_{r}(x)=100\left(1-\hat{\bar{u}}_{r}^{*}(x)\right) .
$$

Finally, the disc-integrated turbulence intensity is defined as

$$
\hat{I}_{r}(x) \simeq \frac{1}{r^{2}} \int_{-r}^{r}|y| I(x, y, 0) d y
$$

One may refer to [1] for further details.

\section{Performance evaluation}

\subsection{Power and thrust coefficients}

First, let us consider the power and thrust coefficients defined with the upstream current velocity $U_{\infty}$. Figure 4 depicts the evolution of the downstream turbine $C_{P, U_{\infty}}^{\text {down }}$ function of its $\operatorname{TSR}_{U_{\infty}}^{\text {down }}$ for different inter-device distances $a^{*}$, compared to the power coefficient $C_{P}$ of one single turbine [1]. The two different ambient intensity rates are considered.

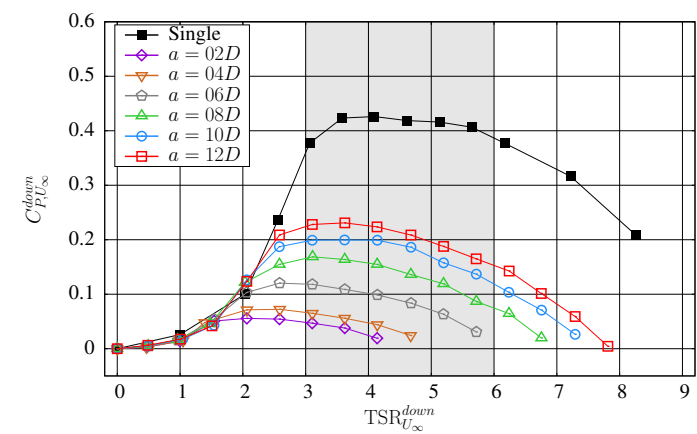

(a) $I_{\infty}=3 \%$

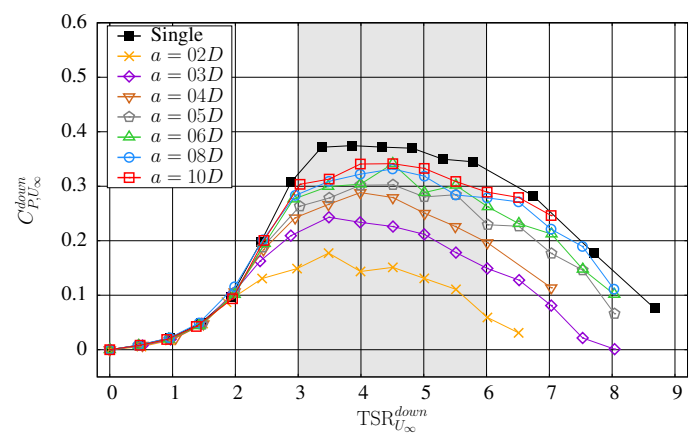

(b) $I_{\infty}=15 \%$

Figure 4: $C_{P, U_{\infty}}^{\text {down }}$ of the downstream device function of its $\operatorname{TSR}_{U_{\infty}}^{\text {down }}$ with $\operatorname{TSR}^{u p}=4, U_{\infty}=0.8 \mathrm{~m} \cdot \mathrm{s}^{-1}, I_{\infty}=3 \%$ (left) and $U_{\infty}=0.83 \mathrm{~m} \cdot \mathrm{s}^{-1}, I_{\infty}=15 \%$ (right), compared to the $C_{P}^{\text {single }}$ of a single turbine shown in Part I [1].

The first striking observation is that, with $I_{\infty}=3 \%$, the maximum downstream $C_{P, U_{\infty}}^{\text {down }}$ is dramatically below the maximum $C_{P}^{\text {single }}$ obtained for one single turbine, even with a $12 D$ interdevice distance. This was expected since the wake behind the single turbine with $I_{\infty}=3 \%$ is still well pronounced even 10 diameters downstream, both in terms of velocity deficit, turbulence intensity and shear stresses. More particularly, there is still a $20 \%$ disc-integrated velocity deficit at this location behind the single turbine, which approximately corresponds to a $50 \%$ deficit of available power [1]. This $50 \%$ deficit in available power is observed here, since the maximum $C_{P, U_{\infty}}^{\text {down }}$ of the downstream turbine with $a=10 D$ is lower than half the maximum $C_{P}^{\text {single }}$ (approximately 0.2 against more than 0.4 ). Second, the operating point seams to decrease with the inter-device distance $\left(\operatorname{TSR}_{U_{\infty}}^{\text {down }} \approx 3.5\right.$ for $a=12 D$, while around $\operatorname{TSR}_{U_{\infty}}^{\text {down }} \approx 2$ for $a=2 D$ ). This is mostly due 
to the fact that the $\operatorname{TSR}_{U_{\infty}}^{\text {down }}$ is defined with the upstream velocity $U_{\infty}$, which is not the velocity that is actually perceived by the turbine, as explained previously in section 2.2.

On the other hand, the $C_{P, U_{\infty}}^{\text {down }}$ evolutions for $I_{\infty}=15 \%$ are more encouraging. Indeed, a similar behaviour to the single turbine is almost recovered from $a=6 D$. From the wake analysis made in Part I [1], this distance corresponds to a recovery of the flow homogeneity (the wake turbulence intensity is similar to its upstream value) together with the lowest velocity deficit (around 5\%). In terms of array implantation, a higher turbulence intensity seems to be much more promising. In fact, to obtain a similar downstream turbine performance, an inter-device distance of 3 diameters is required for $I_{\infty}=15 \%$ to obtain $C_{P, U_{\infty}}^{\text {down }} \approx 0.24$, whereas a $C_{P, U_{\infty}}^{\text {down }} \approx 0.23$ is hardly obtained even with a 12 diameters inter-device distance with $I_{\infty}=3 \%$.

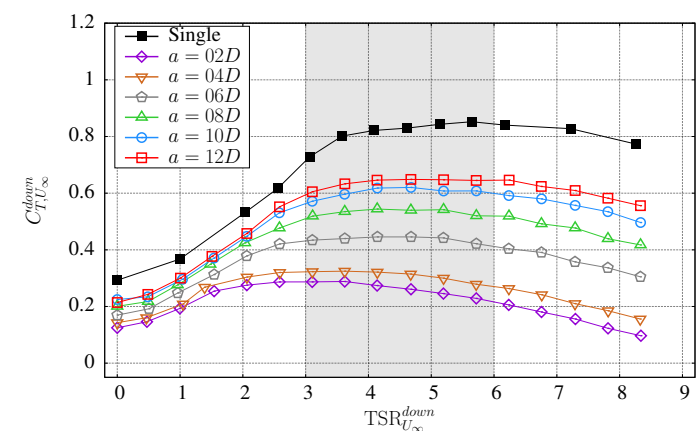

(a) $I_{\infty}=3 \%$

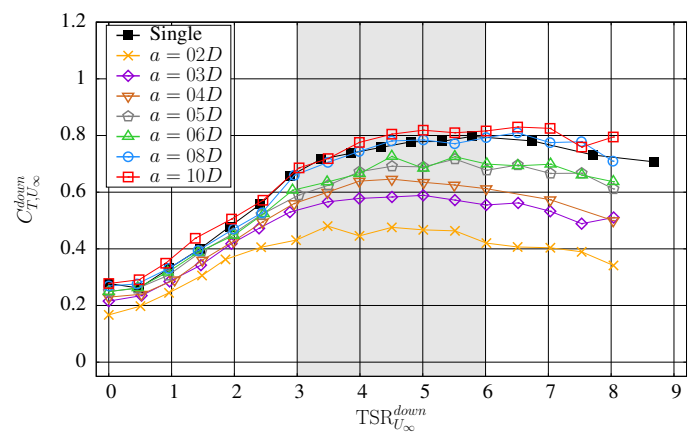

(b) $I_{\infty}=15 \%$

Figure 5: $C_{T, U_{\infty}}^{\text {down }}$ of the downstream device function of its $\operatorname{TSR}_{U_{\infty}}^{\text {down }}$ with $\operatorname{TSR}^{u p}=4, U_{\infty}=0.8 \mathrm{~m} \cdot \mathrm{s}^{-1}, I_{\infty}=3 \%$ (left) and $U_{\infty}=0.83 \mathrm{~m} \cdot \mathrm{s}^{-1}, I_{\infty}=15 \%$ (right), compared to the $C_{T}^{\text {single }}$ of a single turbine shown in Part I [1].

Similar conclusions hold for the downstream turbine thrust, as shows Figure 5. Indeed, the same thrust acts upon the downstream turbine from a $8 D$ inter-device distance as the single turbine with $I_{\infty}=15 \%$. With $I_{\infty}=3 \%$, the thrust always remains below the one measured on the single turbine, even with a $12 D$ distance. In terms of manufacture, this means that both turbines could be designed according to the same thrust baseline.

However, it is important to check that the standard deviations of these coefficients also remain in the same range, since it can influence the device wear and fatigue. Figure 6a shows that the downstream power coefficient standard deviations keep close to the ones of the single turbine, whatever the ambient turbulence intensity is. This corresponds to similar variations in terms of axial moment, but for different operating conditions (i.e. different mean $C_{P}$ ), which might induce a different mechanical behaviour of the turbines. For the thrust standard deviation (Figure 6b), the behaviours are noticeably different depending on the ambient turbulence. In fact, for $I_{\infty}=3 \%$, the thrust standard deviation keeps increasing with the inter-device distance up to $a \approx 8 D$ and then decreases. From the wake analysis of Part I [1], $a \approx 8 D$ corresponds to the end of the shear layers merging $\left(4 \leqslant x^{*} \leqslant 7\right)$ and a maximum of turbulence intensity. It is as if there were a delay in the downstream device reception of the higher turbulence intensity zone created by the wake of the upstream turbine. In the operating range (grey zone, $3 \leqslant \operatorname{TSR}_{U_{\infty}}^{\text {down }} \leqslant 6$ ), the increase of thrust standard deviation may be two to three times higher than the one of a single turbine. This observation may have a crucial influence on the device fatigue for turbines operating in low ambient turbulence intensities. However, if the turbine device is developed based on the $I_{\infty}=15 \%$ thrust 


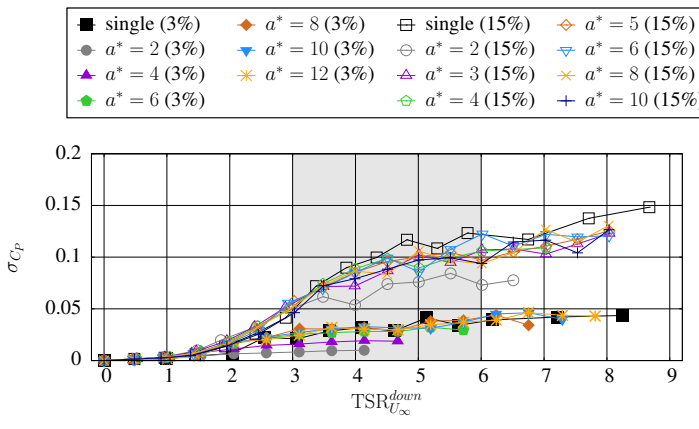

(a) $\sigma_{C_{P}}$

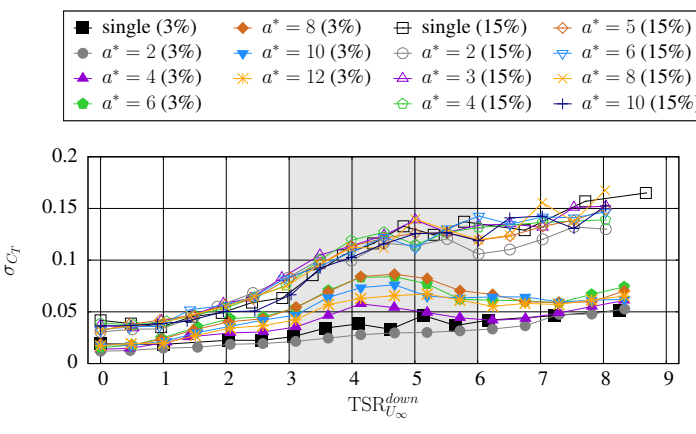

(b) $\sigma_{C_{T}}$

Figure 6: Standard deviation of the downstream turbine $C_{P, U_{\infty}}^{\text {down }}$ (left) and $C_{T, U_{\infty}}^{\text {down }}$ (right) coefficients function of its $\operatorname{TSR}_{U_{\infty}}^{\text {down }}$ with $\mathrm{TSR}^{\text {up }}=4, U_{\infty}=0.8 \mathrm{~m} \cdot \mathrm{s}^{-1}, I_{\infty}=3 \%$ and $U_{\infty}=0.83 \mathrm{~m} \cdot \mathrm{s}^{-1}, I_{\infty}=15 \%$.

standard deviation, this would hardly have an influence since the downstream thrust standard deviations for $I_{\infty}=15 \%$ are very similar to the single turbine, whatever the inter-device distance.

\subsection{Efficiency}

In order to better summarise and visualise the above mentioned information about power collection, an efficiency coefficient $\eta_{u}$, expressed as a percentage, is now defined:

$$
\eta_{u}=100 \frac{\max C_{P, u}^{\text {down }}}{\max C_{P}^{\text {single }}}
$$

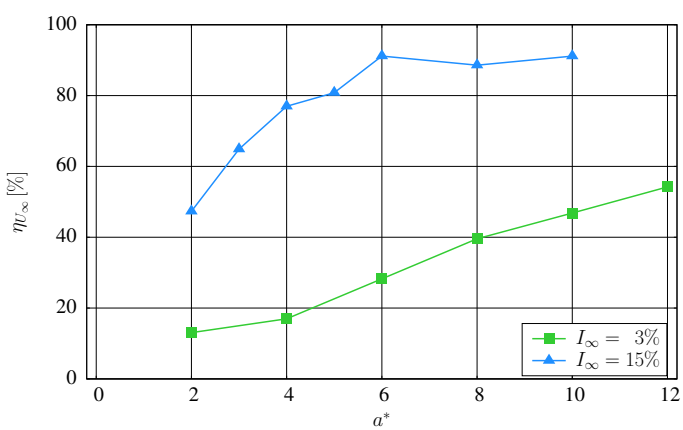

(a) Efficiency $\eta_{U_{\infty}}$

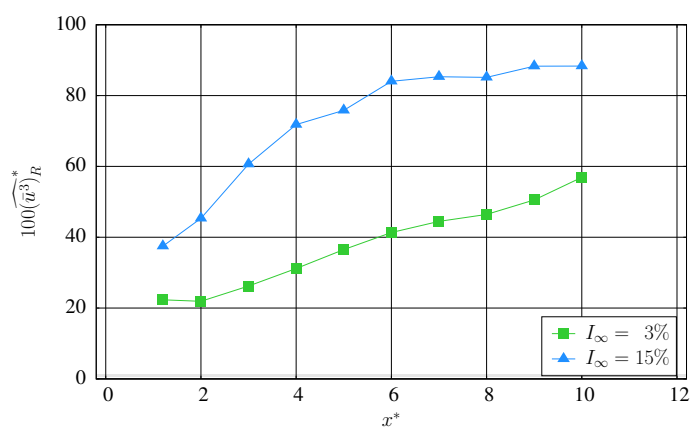

(b) Disc-integrated velocity cubed, $100{\widehat{\left(\bar{u}^{3}\right)}}_{R}^{*}$

Figure 7: (left) Efficiency $\eta_{U_{\infty}}$ of the downstream turbine function of the inter-device distance $a / D$, with $\operatorname{TSR}^{u p}=4$ and (right) evolution of the disc-integrated velocity cubed $\left.\widehat{(\bar{u}}^{3}\right)_{R}^{*}$, expressed as a percentage, behind one single turbine with TSR $=3.67$. Both graphs compare $I_{\infty}=3 \%$ and $15 \%$.

Two main options can then be chosen for $u$, the upstream velocity $U_{\infty}$ or $\hat{\bar{u}}_{R}$, the disc-integrated velocity at the precise location in the wake of the upstream turbine, reproduced from Part I [1]. Let 
first $u$ be set to the upstream velocity $U_{\infty}$. In that case, $\eta_{U_{\infty}}$ then simply represents the percentage of maximum power retrieved by the downstream turbine as compared to one single turbine. On the other hand, one can suppose that the mean velocity integrated on a $R$-radius disc at $x=a, \hat{\bar{u}}_{R}(a)$, is a good indicator of the flow velocity at the location of the downstream turbine.

Figure 7 shows that the $\eta_{U_{\infty}}(a)$ evolution (left) is similar to the evolution of $\left.\widehat{(\bar{u}}^{3}\right)_{R}^{*}(x)=$ $\widehat{\left(\bar{u}^{3}\right)_{R}}(x) / U_{\infty}^{3}$ for the single turbine ${ }^{1}$ (right), for both $I_{\infty}=3 \%$ and $15 \%$. The choice of $U_{\infty}$ for $u$ makes $\eta_{U_{\infty}}$ an indicator of the overall farm efficiency, given a certain upstream flow velocity $U_{\infty}$ that characterises the farm implantation site. Figure 7a presents the $\eta_{U_{\infty}}$ evolution function of the inter-device distance between the two turbines. It represents a translation of Figures $4 \mathrm{a}$ and $4 \mathrm{~b}$ in terms of maximum $C_{P, U_{\infty}}^{\text {down }}$ as compared to a single turbine. This graph gives a more quantitative visualisation of the efficiency evolution depending on the turbulence intensity $I_{\infty}$. As mentioned previously, Figure 7a clearly indicates the very low global efficiency of the second turbine with $I_{\infty}=3 \%$. In addition, this efficiency increases with the inter-device distance but the slope is weak, from $\eta_{U_{\infty}} \approx 15 \%$ at $a^{*}=2$ to a maximum of $\eta_{U_{\infty}} \approx 55 \%$ at $a^{*}=12$. At first glance, this increase seems to follow the trend of $100{\widehat{\left(\bar{u}^{3}\right.}}_{R}^{*}$ as depicted in Figure 7b. Moreover, if the upstream wake keeps the same trend after $12 D$, then a $80 \%$ efficiency should be obtained for at least $a=18 D$. On the contrary, and as mentioned above, with $I_{\infty}=15 \%$ a maximum efficiency of about $90 \%$ seems to be reached from $a=6 D$. The fact that a $100 \%$ efficiency cannot be reached can be explained by the residual $5 \%$ velocity deficit in the far wake of the upstream turbine (for $x^{*} \geqslant 6$, see Part I [1]).

Still considering this efficiency indicator $\eta_{u}$, if $u$ is now set to be the real velocity as it is perceived by the downstream turbine, then $\eta_{u}$ would ideally equal $100 \%$, since the power coefficient of the turbine theoretically only depends on the rotation speed and not on the flow velocity (provided that it is in the turbine operating range, cf. [1]). Thereafter, $\hat{\bar{u}}_{R}(a)$ is chosen to represent the velocity perceived by the downstream turbine. The resulting efficiency $\eta_{\hat{u}_{R}}$ thus characterises the downstream turbine behaviour in relation to the flow conditions existing at its location. Again, the disc integration is performed on $\bar{u}^{3}$ in the computation of $C_{P, \hat{u}_{R}}^{\text {down }}$ and thus of $\eta_{\hat{u}_{R}}$. It should be mentioned that the $\hat{\bar{u}}_{R}$ values are taken from the single turbine study described in Part I [1], for $\mathrm{TSR}=3.67$, while the performance graphs shown here correspond to an upstream $\mathrm{TSR}^{u p}=4$. Nevertheless, the wake behind a turbine with TSR $=3.67$ and TSR $=4$ should be very similar.

Figure 8a shows the evolution of $\eta_{\hat{u}_{R}}$ function of $a$. It is striking to observe that with $I_{\infty}=15 \%$, $\eta_{\hat{\bar{u}}_{R}}$ remains approximately constant and sligthly over $100 \%$. This over-efficiency can be explained either by the fact that a TSR of 3.67 instead of 4 was considered for wake measurements, or by the integral approximation of $\widehat{\left(\bar{u}^{3}\right)_{R}}$ by means of a trapezoid quadrature, which is limited by the number of LDV points on each profile. In addition, the non-homogeneity of the wake makes it difficult to assess the velocity as it is perceived by the turbine. On the other hand, with $I_{\infty}=3 \%$, a $100 \%$ efficiency is never achieved. For short inter-device distances, the downstream turbine is clearly sub-efficient. Then, it increases and a fairly good efficiency of about $85 \%$ is recovered for longer inter-device distances. Actually, three zones can be clearly identified. First of all, for $2 \leqslant a^{*} \leqslant 4$ the efficiency is rather low and constant, with $\eta_{\hat{u}_{R}}$ below $65 \%$. This may be explained by the fact that the perceived velocity is far below the turbine operating range (which starts from $0.6 \mathrm{~m} \cdot \mathrm{s}^{-1}$, see [1]). Indeed, as shows Figure 8b, the assumed flow velocity at the downstream turbine location

\footnotetext{
${ }^{1}$ It should be stressed that the disc integration is performed on the $\bar{u}^{3}$ profiles, thus leading to a ${\widehat{\left(\bar{u}^{3}\right)}}_{R}$ value, which is different from the $\hat{\bar{u}}_{R}^{3}$ value that would be obtained by raising the disc integrated velocity to the cube.
} 


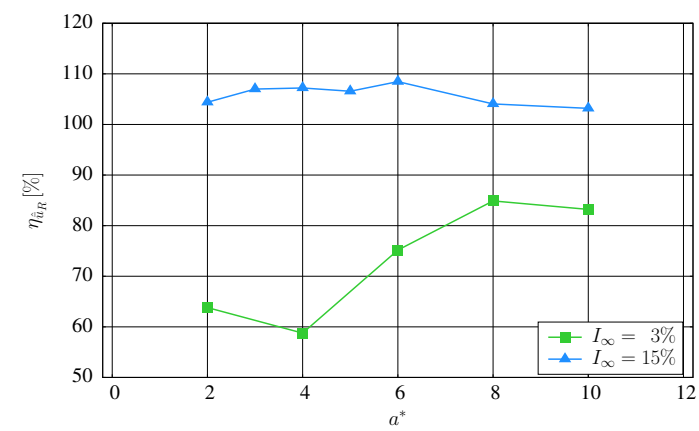

(a) Efficiency $\eta_{\hat{u}_{R}}$

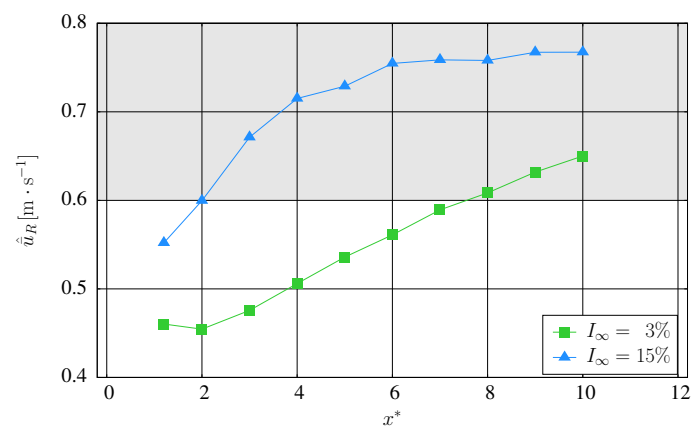

(b) Disc-integrated velocity $\hat{\bar{u}}_{R}$

Figure 8: (left) Efficiency $\eta_{\hat{\bar{u}}_{R}}$ of the downstream turbine function of the inter-device distance $a / D$, with TSR ${ }^{u p}=4$ and (right) evolution of the dimensional disc-integrated velocity $\hat{\bar{u}}_{R}$ (in $\mathrm{m} \cdot \mathrm{s}^{-1}$ ), behind one single turbine with $\mathrm{TSR}=3.67$. The assumed operating range, in terms of velocity, is highlighted by the grey zone $\left(\geq 0.6 \mathrm{~m} \cdot \mathrm{s}^{-1}\right)$. Both graphs compare $I_{\infty}=3 \%$ and $15 \%$.

remains far below $0.6 \mathrm{~m} \cdot \mathrm{s}^{-1}$ for the considered distances. Moreover, the wake flow behind the upstream turbine is driven by the rotation, which is an additional factor penalising the downstream turbine performance independently of the reduced flow velocity. The second zone corresponds to $4<a^{*}<8$. In this area, the perceived velocity is still below $0.6 \mathrm{~m} \cdot \mathrm{s}^{-1}$ but keeps increasing towards the operating range (grey zone in Fig. 8b). In addition, this zone was clearly identified in [1] as the merging zone where the shear layers begin to mix. The flow then becomes more and more homogeneous, which accounts for the efficiency increase. Eventually, from $a^{*} \geqslant 8$, the maximum efficiency seems to be reached. The perceived velocity is then in the operating range (Fig. 8b) and the flow has recovered most of its homogeneity [1]. The little sub-efficiency $\left(\eta_{\hat{u}_{R}} \approx 85 \%\right)$ might be attributed to the presence of residual coherent vortical structures even $10 D$ downstream of the first turbine. In fact, as discussed in Part I [1, section Wake characterisation], the upstream turbulence intensity conditions of $I_{\infty}=3 \%$ are never completely recovered, even 10 diameters behind the first turbine.

From the analysis described above, several conclusions can be drawn. Firstly, the downstream performances increase with the inter-device distance $a$, whatever the ambient turbulence intensity, $I_{\infty}=3 \%$ or $15 \%$. The local fluid velocity, at the location of the downstream turbine, is the major influencing parameter (Fig. 7). However, regarding the efficiency $\eta_{\hat{u}_{R}}$ based on this local velocity (Fig. 8), a $100 \%$ efficiency is not always obtained. In fact, the flow homogeneity is, to the authors' opinion, the second major influencing parameter. In the near wake of a turbine, where the wake flow is well characterised for $I_{\infty}=3 \%$, this $\eta_{\hat{u}_{R}}$ efficiency is dramatically reduced. For $I_{\infty}=3 \%$, this $\eta_{\hat{u}_{R}}$ efficiency increases with the homogeneity recovery, but still does not reach $100 \%$ since the downstream turbulence intensity has not recovered its far upstream conditions, even for large inter-device distances. However, from the single turbine study [1], the higher the ambient turbulent intensity, the faster the homogeneity is recovered in the wake flow. 


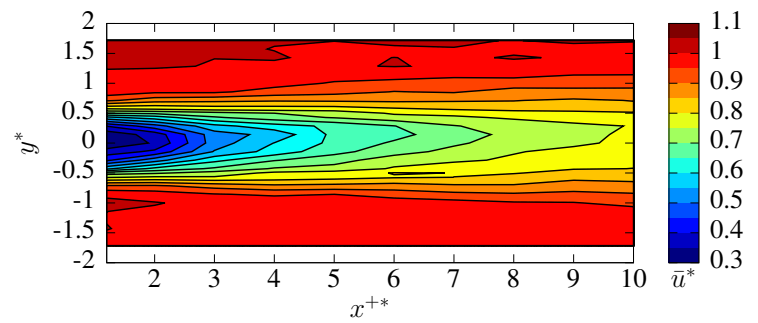

(a) Axial velocity map $\left(I_{\infty}=3 \%\right)$

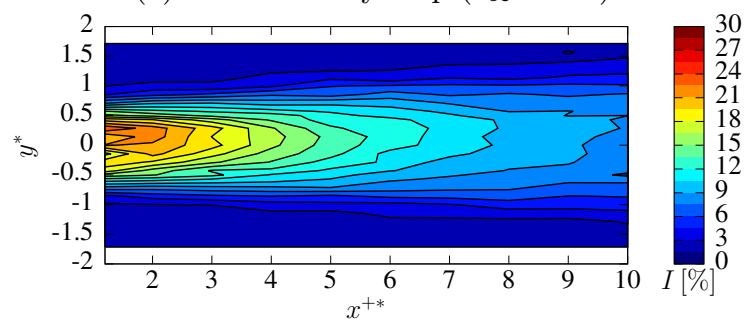

(c) Turbulence intensity map $\left(I_{\infty}=3 \%\right)$

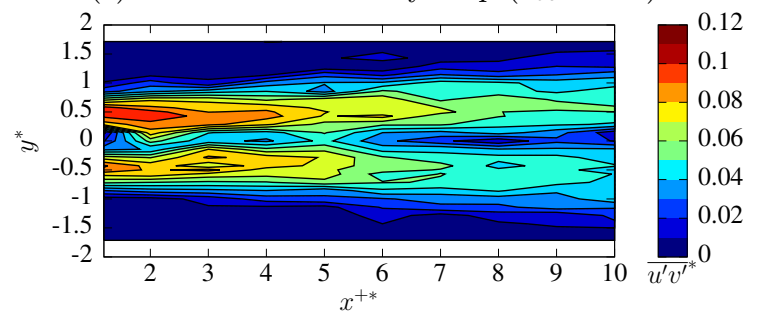

(e) Reynolds shear stress map $\left(I_{\infty}=3 \%\right)$

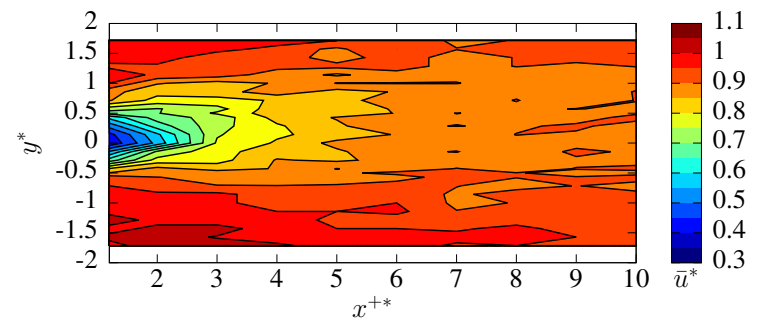

(b) Axial velocity map $\left(I_{\infty}=15 \%\right)$

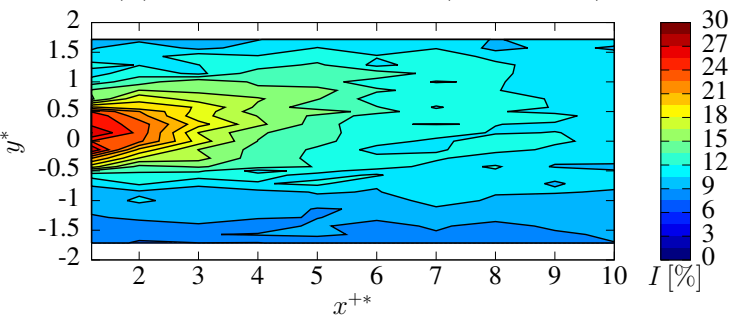

(d) Turbulence intensity map $\left(I_{\infty}=15 \%\right)$

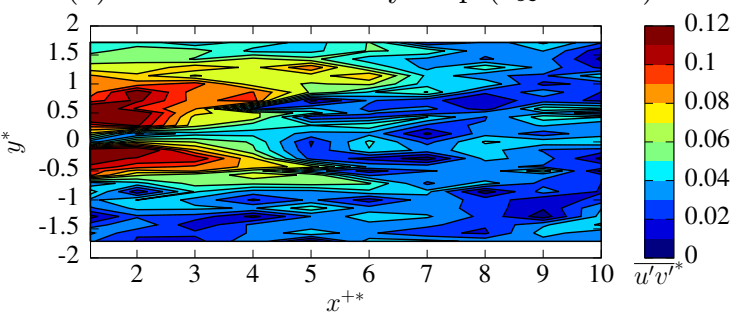

(f) Reynolds shear stress map $\left(I_{\infty}=15 \%\right)$

Figure 9: Wake behind the downstream turbine with $a=4 D$, and $\operatorname{TSR}^{u p}=\operatorname{TSR}_{U_{\infty}}^{\text {down }}=3.67$.

\section{Wake characterisation}

The wake behind the downstream turbine may also be altered by the influence of the upstream turbine. Indeed, depending on the inter-device distance, the flow at the location of the downstream turbine may not be homogeneous and may suffer as well from a velocity deficit as compared to the array upstream velocity $U_{\infty}$. Figure 9 presents axial velocity, turbulence intensity and Reynolds shear stress intensity maps behind the downstream turbine. The turbines are spaced by $a^{*}=4$ and both of them are rotating at the same speed such that $\mathrm{TSR}^{u p}=\mathrm{TSR}_{U_{\infty}}^{\text {down }}=3.67$. Similarly to the single turbine wake [1], major differences can be observed between $I_{\infty}=3 \%$ maps and $I_{\infty}=15 \%$ maps.

With $I_{\infty}=3 \%$, the downstream velocity deficit still remains contained in an axial strip, which is slightly larger than the one behind a single turbine. The wake also seems to recover slightly faster in terms of velocity deficit. However, a major difference from the results on one single turbine resides in the turbulence intensity and shear stress maps. Indeed, while the merging zone behind one single turbine is located around $5 \leqslant x^{*} \leqslant 7$, it is located in the very near wake of the downstream turbine. This confirms that the flow may recover its homogeneity faster. This is mainly due to the higher turbulence intensity at the location of the downstream turbine, $\hat{I}_{R}$ being around $12 \%$ 
instead of $3 \%$ (cf. Figure 11c, single turbine curve). In that manner, Figures 9a, 9c and 9e can be viewed as an intermediary between a single turbine immersed in a $I_{\infty}=3 \%$ and $I_{\infty}=15 \%$. As regards $I_{\infty}=15 \%$, the wakes are very similar to those of one single turbine, even in terms of shear stress. This indicates that the flow characteristics seem to be hardly altered by the presence of the upstream turbine, or at least that these characteristics are recovered very shortly behind the upstream turbine (i.e. before $4 D$ ).

It is worth mentioning that wake measurements behind the downstream turbine were performed for other distances. For $I_{\infty}=3 \%$, configurations with $a^{*}=4 ; 6 ; 8 ; 10$ were considered, while for $I_{\infty}=15 \%$ configurations with $a^{*}=2 ; 4 ; 6$ were run. It was striking to realise that the wake maps were extremely alike, as a consequence, these maps are not reproduced here. Nevertheless, still with a view to encourage future comparisons, all of our raw results are made available in Appendix A, as downstream velocity and turbulence intensity profiles.

The wake behaviour can be translated in terms of disc-integrated quantities [1]. Figure 10 translates the maps from Figure 9 into axial velocity deficit and downstream turbulence intensity evolution function of the distance from the downstream turbine. Centreline evolutions are compared to disc-integrated evolutions.

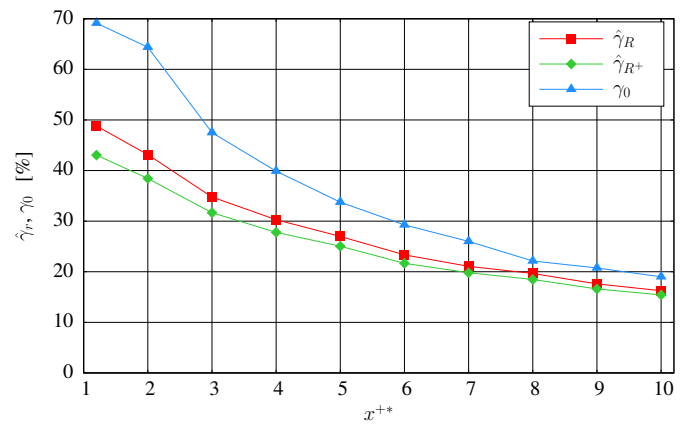

(a) Axial velocity deficit $\left(I_{\infty}=3 \%\right)$

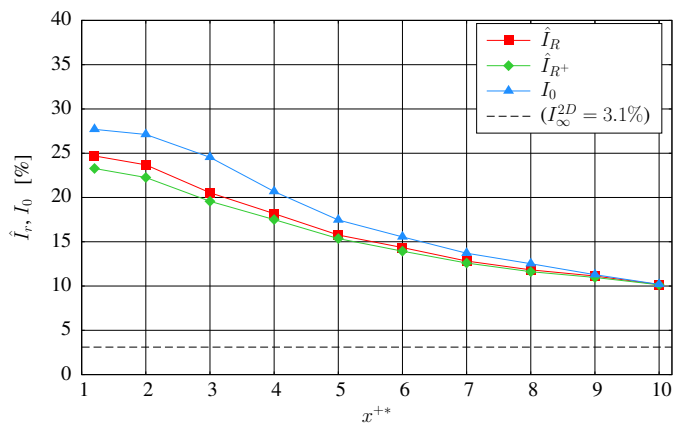

(c) Turbulence intensity $\left(I_{\infty}=3 \%, I_{\infty}^{2 D} \approx 3.1 \%\right)$

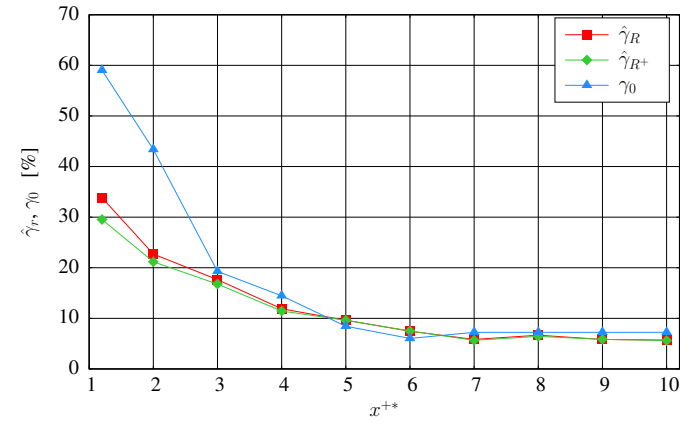

(b) Axial velocity deficit $\left(I_{\infty}=15 \%\right)$

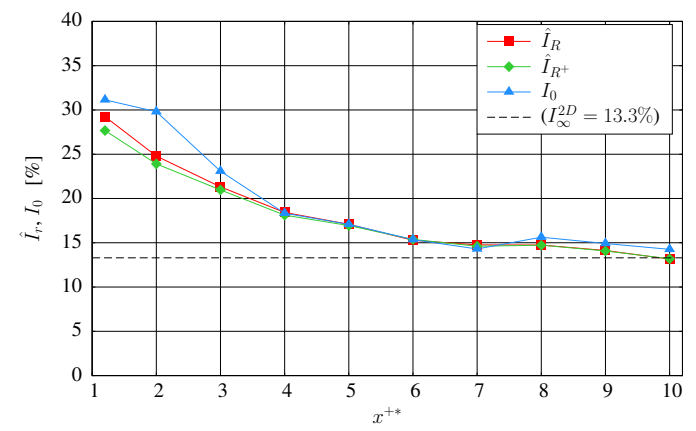

(d) Turbulence intensity $\left(I_{\infty}=15 \%, I_{\infty}^{2 D} \approx 13.3 \%\right)$

Figure 10: Velocity deficit (top) and turbulence intensity (bottom) in the wake of the downstream turbine with $a=4 D, \mathrm{TSR}^{u p}=\mathrm{TSR}_{U_{\infty}}^{\text {down }}=3.67$, for $I_{\infty}=3 \%$ (left) and $15 \%$ (right).

The previously mentioned conclusions are confirmed. For $I_{\infty}=3 \%$, the disc-integrated velocity deficit $\hat{\gamma}_{R}$, ten diameters downstream, is slightly lower than for the single turbine. The integrated 
quantities $\left(\hat{\gamma}_{R}, \hat{\gamma}_{R^{+}}, \hat{I}_{R}\right.$ and $\left.\hat{I}_{R^{+}}\right)$are much closer to their centreline counterpart, which is not the case with one single turbine. This tends to indicate that the wake effect of the downstream turbine immersed in a $I_{\infty}=3 \%$ ambient turbulence intensity is less pronounced than the one of a single turbine. On the contrary, for $I_{\infty}=15 \%$, the evolution of all these quantities (centreline or disc-integrated) are basically similar to the single turbine case (see Figures 11b and 11d single turbine curves).

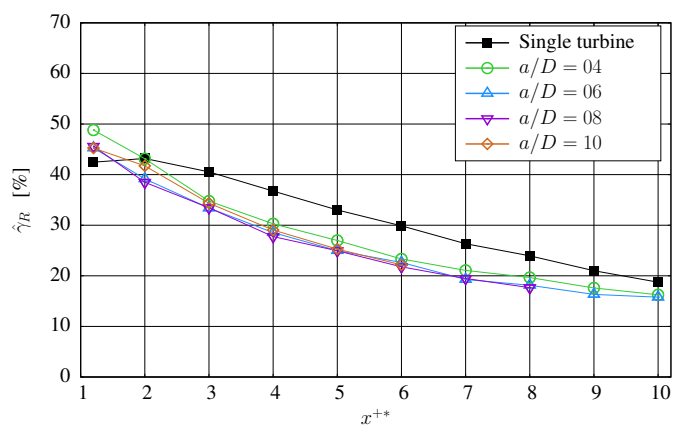

(a) Velocity deficit $\left(I_{\infty}=3 \%\right)$

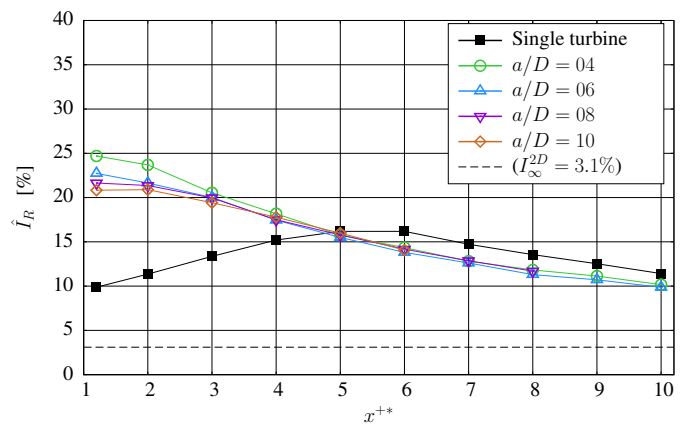

(c) Turbulence intensity $\left(I_{\infty}=3 \%, I_{\infty}^{2 D} \approx 3.1 \%\right)$

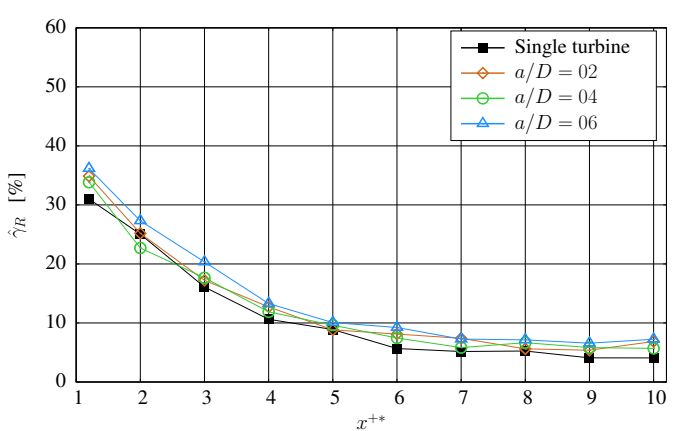

(b) Velocity deficit $\left(I_{\infty}=15 \%\right)$

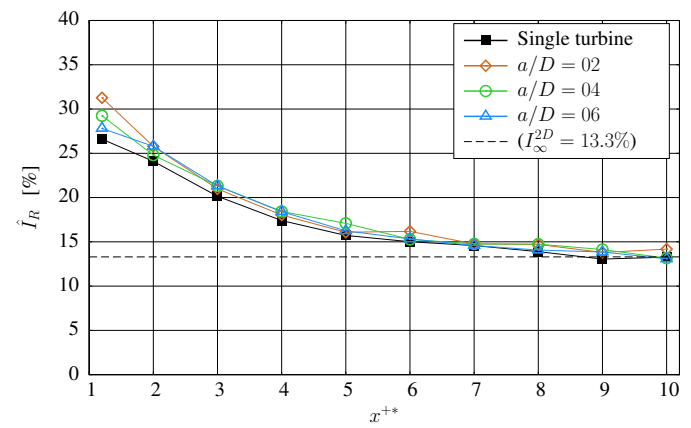

(d) Turbulence intensity $\left(I_{\infty}=15 \%, I_{\infty}^{2 D} \approx 13.3 \%\right)$

Figure 11: Mean disc-integrated velocity deficit $\hat{\gamma}_{R}$ (top) and turbulence intensity $\hat{I}_{R}$ (bottom) in the wake of the downstream turbine with for various distances $a / D$ between the devices, $\operatorname{TSR}^{u p}=\operatorname{TSR}_{U_{\infty}}^{\text {down }}=3.67$, for $I_{\infty}=3 \%$ (left) and $15 \%$ (right).

In order to better visualise the influence of the inter-device distance $a$ on the downstream wake evolution, the disc-integrated velocity deficit $\hat{\gamma}_{R}$ and turbulence intensity $\hat{I}_{R}$ are presented in Figure 11 for different values of $a$. The first striking observation is that, for both turbulence intensities $I_{\infty}$, the wake shape and evolution behind the downstream turbine are hardly influenced by the inter-device distance $a$. This means that whatever the velocity deficit induced by the presence of the upstream turbine, the downstream turbine will behave such that it causes a similar velocity deficit evolution in its wake.

Secondly, for $I_{\infty}=3 \%$, as mentioned previously, the wake evolution is slightly different from one single turbine. More particularly, the velocity deficit recovery rate gets closer to a $I_{\infty}=15 \%$ evolution, instead of an almost linear low decrease, characteristic of the single $I_{\infty}=3 \%$ case. The difference is even more noticeable on the turbulence intensity evolution. Indeed, the highest 
turbulence intensity area is located directly behind the turbine, contrary to the single turbine case. This can again be related to the shear layers merging zone location, which is different in those two cases. On the contrary, as expected from the previous observations, the wake evolution curves with $I_{\infty}=15 \%$ are almost identical to the one of the single turbine configuration.

To conclude, these results provide valuable information for array implantation. They tend to indicate that the distance between downstream turbines may be equally repeated without causing any different wake behaviour, which may hopefully result in similar performances for all the turbines. The fact that, for each of the considered turbulence intensity rates, the second row turbine wakes are very similar whatever the inter-device distance is very promising. This is even more encouraging as our two ambient turbulence intensities are very different and seem to cover the real condition range of ambient turbulence intensities (see Part I [1, Introduction]). However for potential sites with a low ambient turbulence intensity, the first inter-device distance (i.e. between the very upstream and the second turbine) may need to be different. This is particularly true in our $I_{\infty}=3 \%$ case, where the wake behind the first turbine is noticeably different. Further trials with three aligned turbines could represent an interesting study case to check the above mentioned assumptions.

\section{Conclusions and prospects}

Single configurations data [1] were used as a basis to carry out trials on two devices configurations aligned with the flow in this second part of the study. Both wake and performance analysis were characterised, qualitatively and quantitatively, with a large range of inter-device spacings, up to 12 diameters between the two turbines. All configurations were tested with two different ambient turbulence intensities, namely $I_{\infty}=3 \%$ and $I_{\infty}=15 \%$, which seems to mark out the boundaries of the real condition values [1]. In this aligned configuration, wake interaction effects between the turbines exist; the downstream turbine may thus be deeply affected by the presence of an upstream device. Moreover, the ambient turbulence intensity plays a major role in the different behaviours of the presented configurations.

Concerning performances, the higher the ambient turbulence intensity, the better the performances of the downstream turbine for a given inter-device distance. For $I_{\infty}=15 \%$, a global efficiency of $\eta_{U_{\infty}} \approx 90 \%$ is reached from a $6 D$ inter-device distance, whereas $\eta_{U_{\infty}} \approx 50 \%$ is hardly obtained with a $12 D$ spacing for $I_{\infty}=3 \%$. As expected, the thrust coefficient curves are slightly lower than the single turbine ones for $I_{\infty}=3 \%$ and they tend to superimposed as the inter-device distance increases for $I_{\infty}=15 \%$. From a structural fatigue point of view, there is a major difference between the two $I_{\infty}$ levels as, in the operating range of the turbine, the standard deviations on the thrust coefficient is always two to three times higher with $I_{\infty}=15 \%$ than with $I_{\infty}=3 \%$. The standard deviations of both the power and thrust coefficients tend to superimposed with their single turbine counterparts, whatever the ambient turbulence intensity, the only exception being the thrust coefficient standard deviation for $I_{\infty}=3 \%$. However, it should be stressed that similar standard deviations for different operating conditions might imply different mechanical behaviours in terms of fatigue. On the other hand, regarding the $\eta_{\hat{u}_{R}}$ curves (the turbine efficiency computed with the local velocity), this value is always close to $100 \%$ for $I_{\infty}=15 \%$. For the configuration with a $3 \%$ ambient turbulence intensity, the wake effects are much more complex leading to a more complex curve which hardly reaches $85 \%$ for a $10 D$ inter-device distance.

As far as the wakes are concerned, one of the major advantages of the higher ambient turbulent case is that the wakes of the upstream and downstream turbines are much alike. The other major advantage resides in the fact that, whatever the upstream or downstream turbine wake, the flow 
recovers its far upstream ambient turbulence intensity of 15\% from 6 diameters behind the device together with approximately $10 \%$ decreasing to $5 \%$ velocity deficit. This behaviour is consistent whatever the inter-device distance of the two turbines $(a / D=2$ to 6 were tested). For the $3 \%$ ambient turbulence intensity conditions, the upstream and downstream turbines wakes are rather different. In addition, the far upstream ambient turbulence intensity $I_{\infty}=3 \%$ is never recovered with up to 3 times its value at 10 diameters downstream. However, the behaviour of the downstream wake is also consistent whatever the inter-device distance of the two turbines $(a / D=4$ to 10 were tested). This can be viewed as an interesting feature for the trial configuration of three turbines aligned with the flow, which might represent one of our future studies.

For industrial applications, the numerical modelling of the array global performance will then be easier for sites with high turbulence intensity, as the efficiency $\eta_{\hat{\bar{u}}_{R}} \approx 100 \%$ is a valid assumption (for $I_{\infty}=15 \%$ for instance on Fig. 8). The knowledge of the local velocity in the array will then give an accurate performance assessment of a given turbine, even in the second or third row. Lower ambient turbulence intensity will damage the accuracy of this $\eta_{\hat{\bar{u}}_{R}} \approx 100 \%$ assumption leading to a more complex global performance assessment.

For these lower ambient turbulence intensity sites, represented in the current case with $I_{\infty}=3 \%$, the performances of the turbines second row will be dramatically reduced unless very large interdevice distances are chosen (i.e. $\eta_{U_{\infty}} \approx 50 \%$ of the nominal performance at 12 diameters). Another possibility would be to choose a lower TSR for the first row. In fact, it was pointed out in [18] that the downstream device can retrieve more power when the upstream turbine has its TSR $=3$ rather than 4 . These results indicate that a compromise between individual performances and global farm performances can be found by optimising inter-device spacing. Considering a farm implantation in a given area (bathymetry, depth, flow characteristics), the more distant two successive rows of turbines are, the higher the individual power retrieved is. But there is then less space for additional rows of devices, so fewer turbines can be implanted. It is clear that increasing inter-device spacing to retrieve higher individual power can only be done to the detriment of the total number of turbine rows in a given space. So a compromise between individual performance and the number of energy converters has to be made wisely when considering an array implantation.

A single turbine geometry was tested, a three-bladed horizontal axis turbine and hence the presented results may differ with other turbine geometries (blade shape, two-bladed turbine). However, a detailed description of the turbine geometry is given as well as all our raw results in the aim of future comparisons with numerical computations. Some of our prospects, which concern both the experimental and the numerical aspect, consist in modelling other kinds of turbine prototypes or in taking into account both wave and current effects on the behaviour of marine current turbines. Other ambient turbulence intensities between $I_{\infty}=3 \%$ and $I_{\infty}=15 \%$ may also be tested. 


\section{Appendix A. Wake profiles}

Appendix A.1. Profiles with $I_{\infty}=3 \%$

$$
\begin{aligned}
& \text { - } x^{+*}=1.2 \quad \text { ष } x^{+*}=6.0 \\
& \leadsto x^{+*}=2.0 \leadsto x^{+*}=7.0 \\
& \triangle x^{+*}=3.0 \leadsto x^{+*}=8.0
\end{aligned}
$$

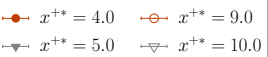

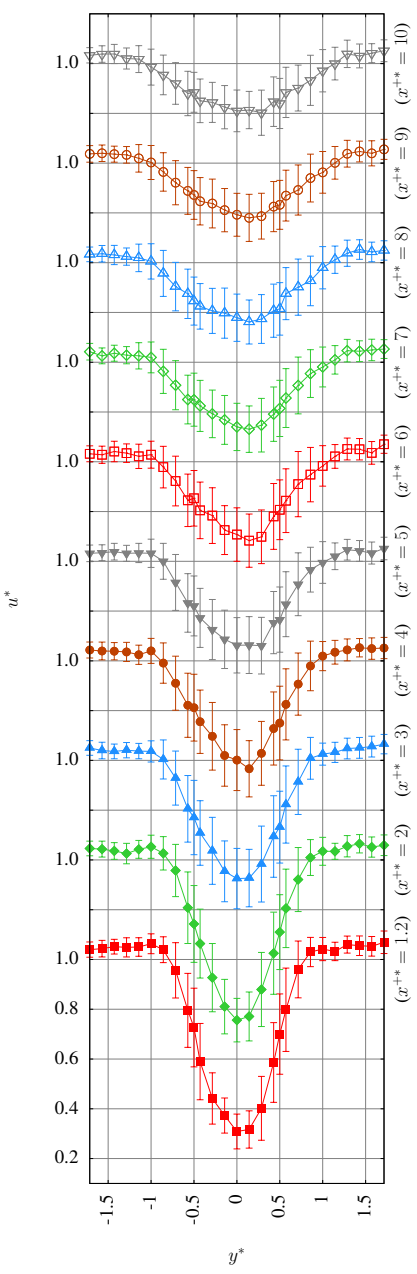

(a) $a / D=4$
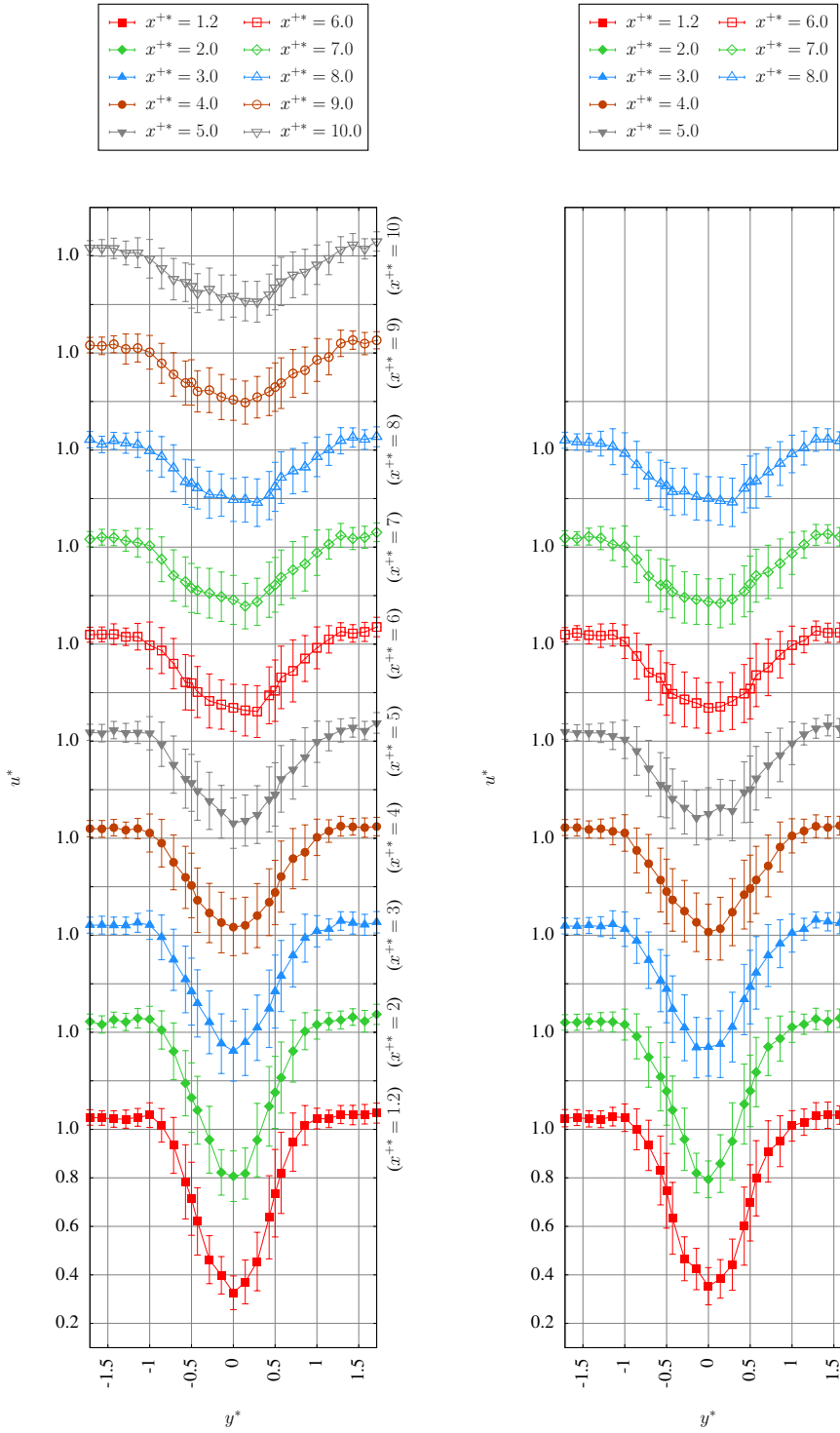

(b) $a / D=6$

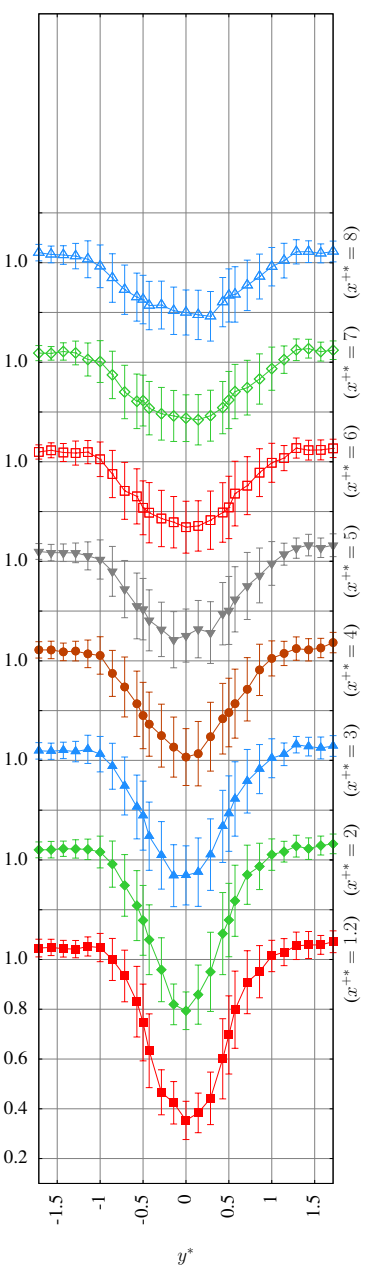

(c) $a / D=8$

Figure A.12: Axial velocity profiles with $\mathrm{TSR}^{u p}=\mathrm{TSR}^{\text {down }}=3.67, U_{\infty}=0.8 \mathrm{~m} \cdot \mathrm{s}^{-1}, I_{\infty}=3 \%$ and with $a^{*}=4$ (left), $a^{*}=6$ (middle) and $a^{*}=8$ (right). 

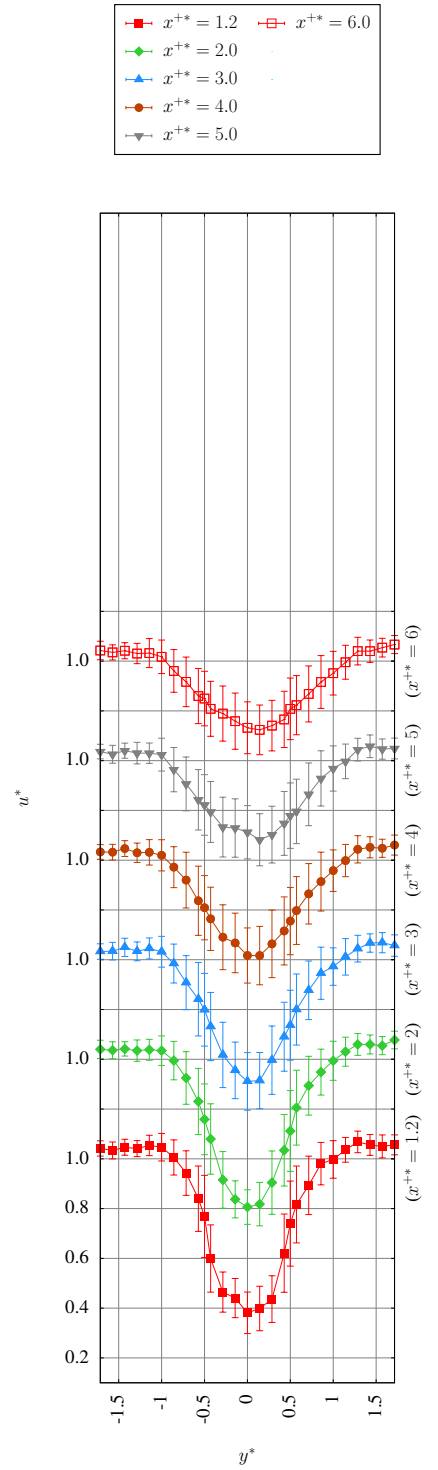

Figure A.13: Axial velocity profiles with $\mathrm{TSR}^{u p}=\mathrm{TSR}^{\text {down }}=3.67, U_{\infty}=0.8 \mathrm{~m} \cdot \mathrm{s}^{-1}, I_{\infty}=3 \%$ and with $a^{*}=10$. 


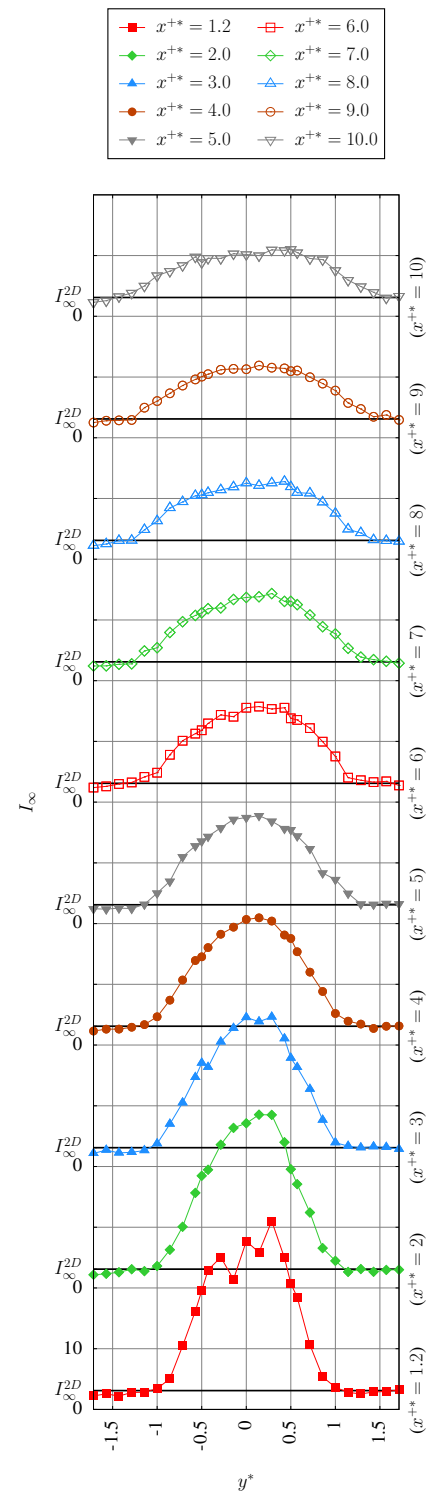

(a) $a / D=4$
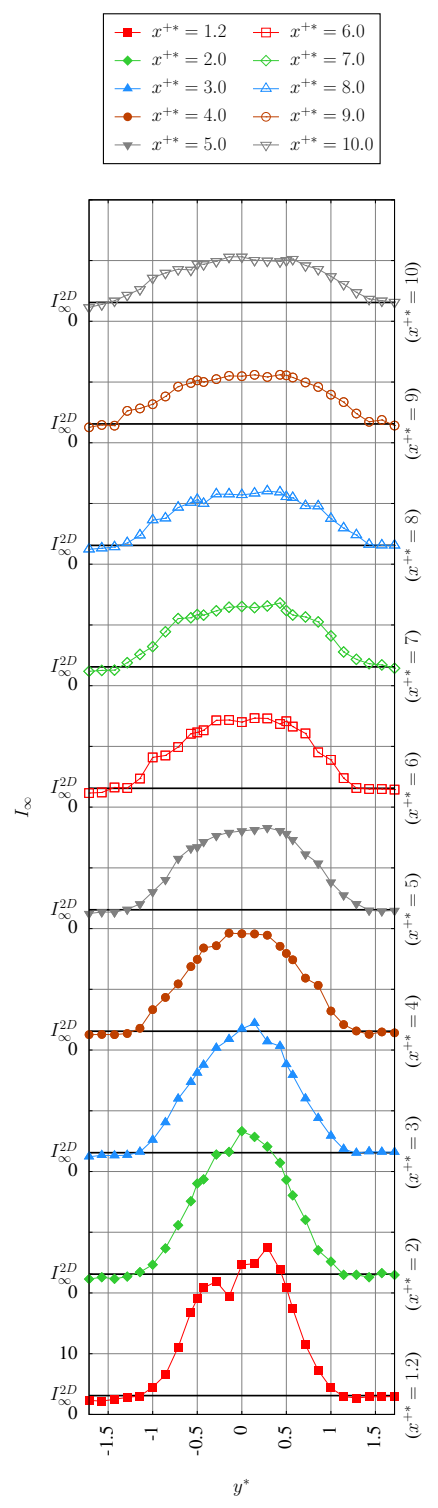

(b) $a / D=6$
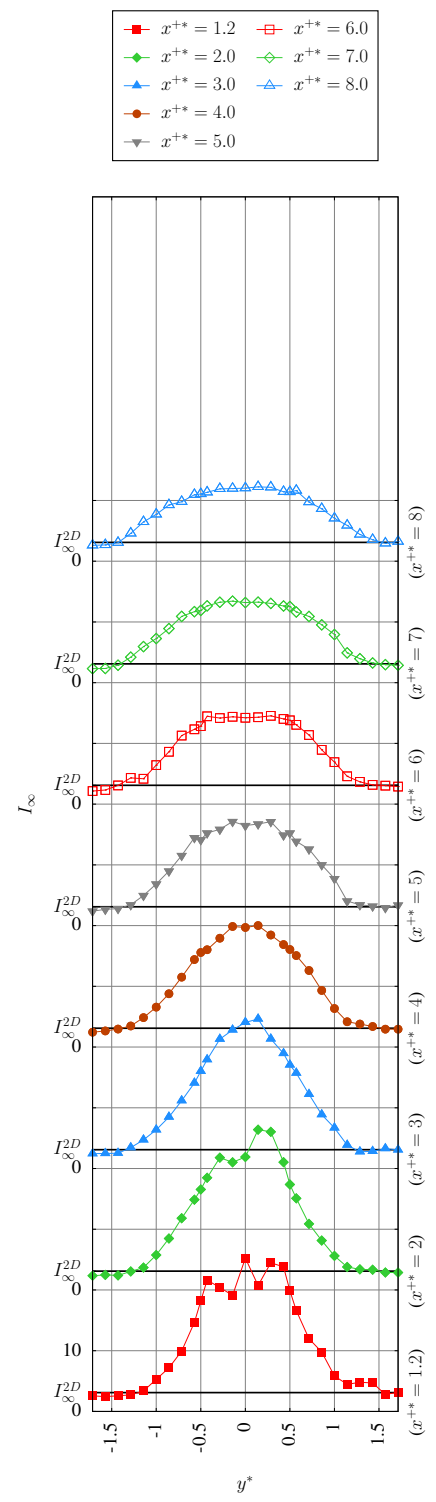

(c) $a / D=8$

Figure A.14: Downstream turbulence intensity profiles with $\mathrm{TSR}^{u p}=\mathrm{TSR}^{\text {down }}=3.67, U_{\infty}=0.8 \mathrm{~m} \cdot \mathrm{s}^{-1}, I_{\infty}=3 \%$ and with $a^{*}=4$ (left), $a^{*}=6$ (middle) and $a^{*}=8$ (right). 


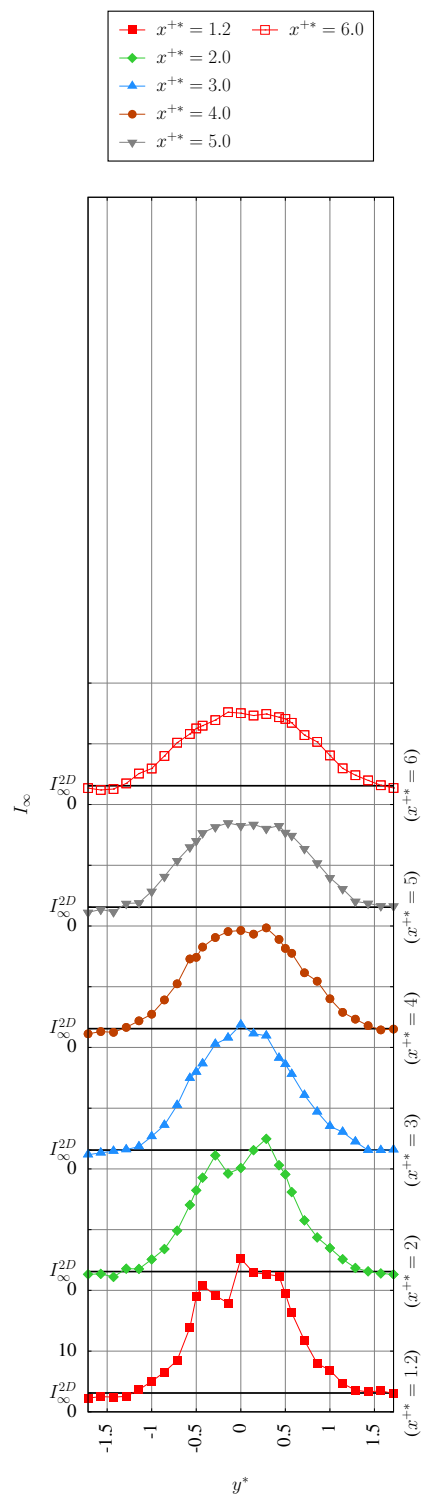

Figure A.15: Downstream turbulence intensity profiles with $\mathrm{TSR}^{u p}=\mathrm{TSR}^{\text {down }}=3.67, U_{\infty}=0.8 \mathrm{~m} \cdot \mathrm{s}^{-1}, I_{\infty}=3 \%$ and with $a^{*}=10$. 
Appendix A.2. Profiles with $I_{\infty}=15 \%$

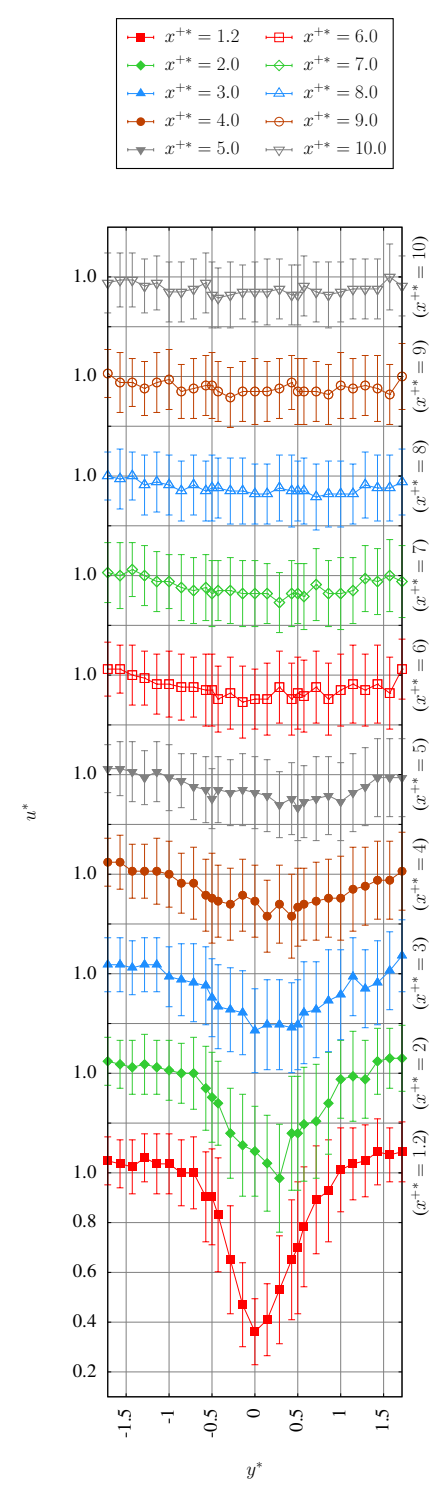

(a) $a / D=2$
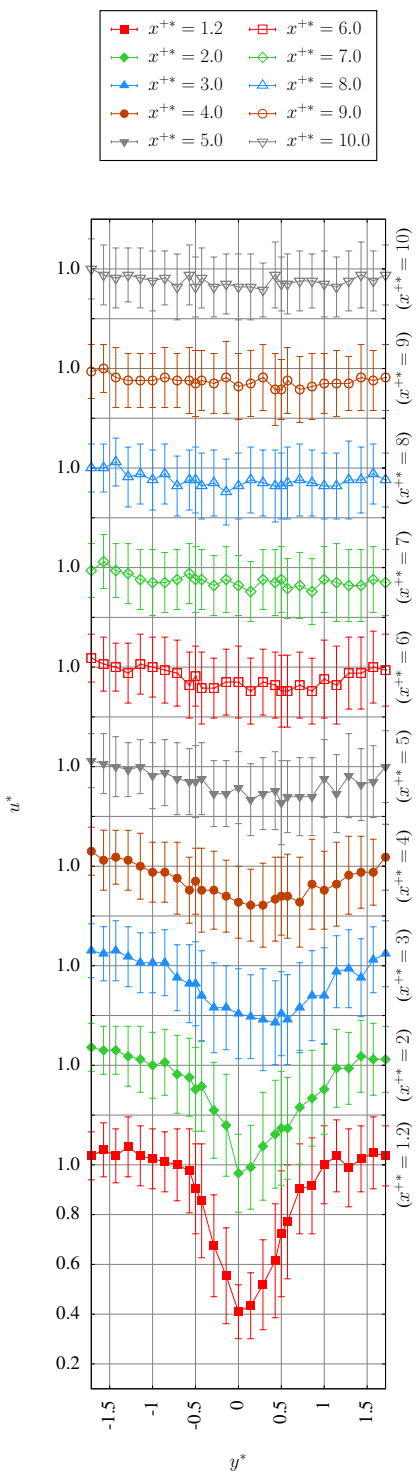

(b) $a / D=4$
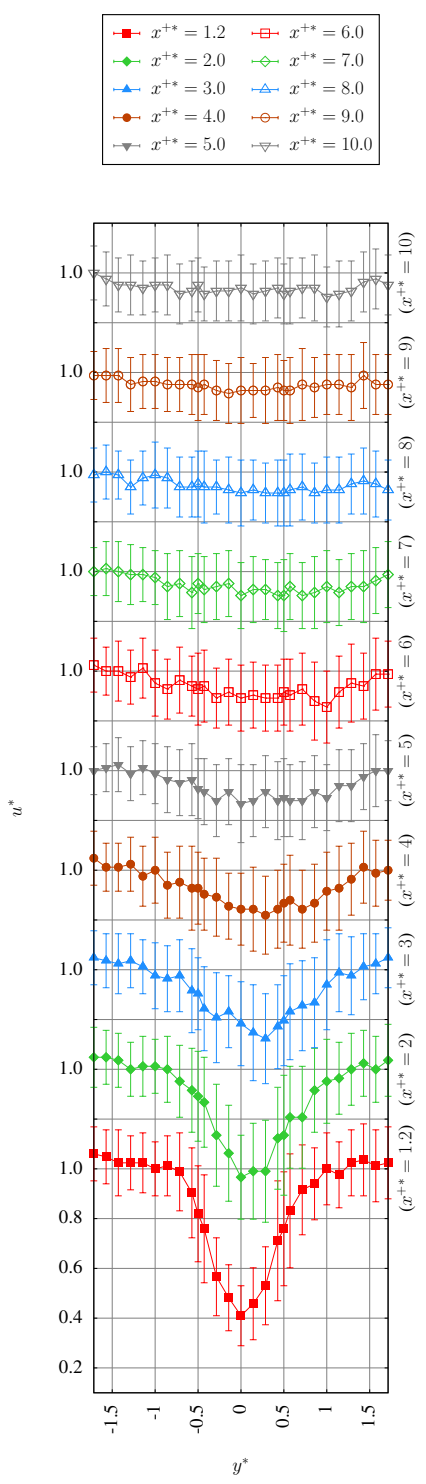

(c) $a / D=6$

Figure A.16: Axial velocity profiles with $\mathrm{TSR}^{u p}=\mathrm{TSR}^{\text {down }}=3.67, U_{\infty}=0.8 \mathrm{~m} \cdot \mathrm{s}^{-1}, I_{\infty}=15 \%$ and with $a^{*}=2$ (left), $a^{*}=4$ (middle) and $a^{*}=6$ (right). 


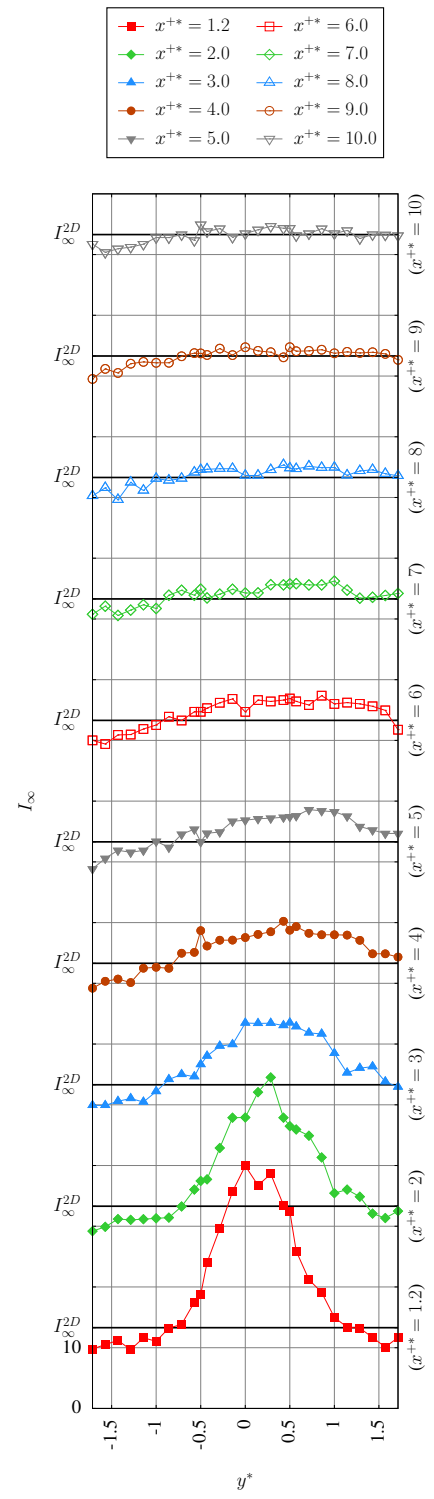

(a) $a / D=2$

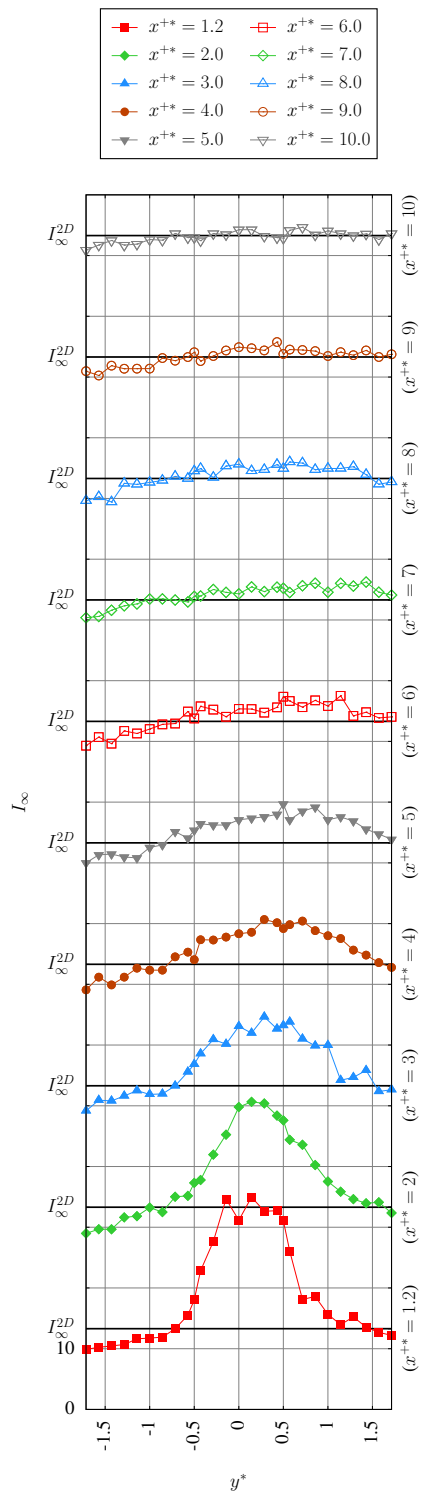

(b) $a / D=4$
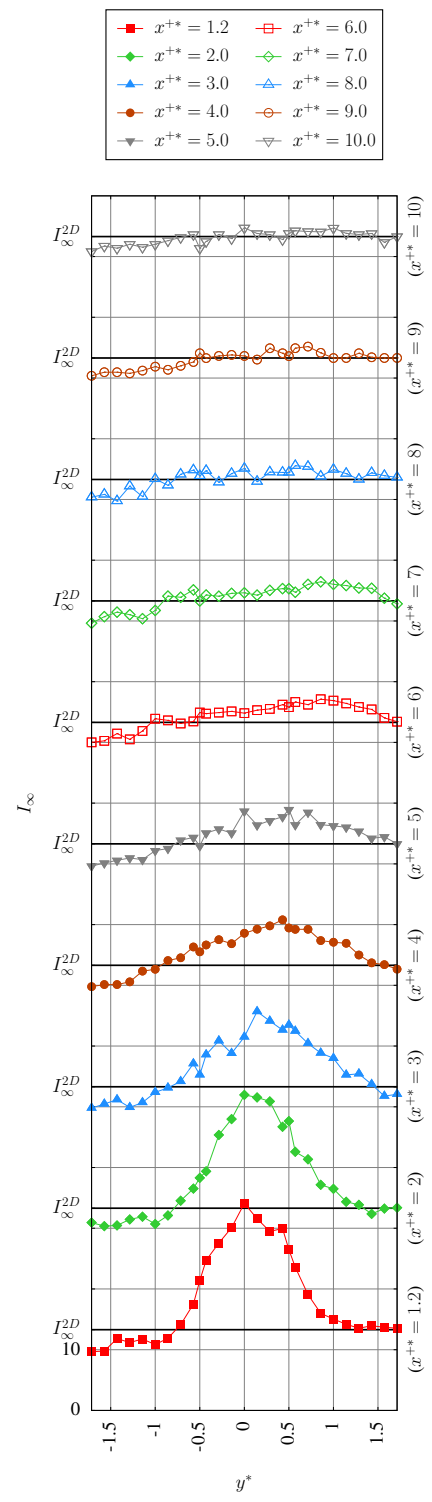

(c) $a / D=6$

Figure A.17: Downstream turbulence intensity profiles with $\mathrm{TSR}^{u p}=\mathrm{TSR}^{\text {down }}=3.67, U_{\infty}=0.8 \mathrm{~m} \cdot \mathrm{s}^{-1}, I_{\infty}=15 \%$ and with $a^{*}=2$ (left), $a^{*}=4$ (middle) and $a^{*}=6$ (right). 


\section{Acknowledgments}

The authors would like to thank Haute-Normandie Regional Council and IFREMER for their financial support of co-financed PhD theses, as well as the RHYNO project. We are most grateful to Thomas Bacchetti and Jean-Valery Facq for their help in the present work.

\section{References}

[1] P. Mycek, B. Gaurier, G. Germain, G. Pinon, E. Rivoalen, Experimental study of the turbulence intensity effects on marine current turbines behaviour. Part I: one single turbine, Submitted to Renewable Energy (2013).

[2] F. Maganga, G. Germain, J. King, G. Pinon, E. Rivoalen, Experimental characterisation of flow effects on marine current turbine behaviour and on its wake properties, IET Renewable Power Generation 4 (2010) 498-509.

[3] S. J. Couch, H. F. Jeffrey, Tidal current energy: Further development of protocols for equitable testing of device performance, in: 3rd International Conference on Ocean Energy, 2010.

[4] R. Rawlinson-Smith, I. Bryden, M. Folley, V. Martin, T. Stallard, C. Stock-Williams, R. Willden, The perawat project: Performance assessment of wave and tidal array systems, in: 3rd International Conference on Ocean Energy, 2010.

[5] L. Myers, A. Bahaj, Experimental analysis of the flow field around horizontal axis tidal turbines by use of scale mesh disk rotor simulators, Ocean Engineering 37 (2010) 218-227.

[6] C.-H. Jo, J.-Y. Yim, K.-H. Lee, K.-O. Ko, Performance of multi-arrayed tidal current power rotors, in: 3rd International Conference on Ocean Energy (ICOE), 2010. Bilbao, Spain.

[7] T. Stallard, R. Collings, T. Feng, J. Whelan, Interactions between tidal turbine wakes: experimental study of a group of 3-bladed rotors, in: 9th European Wave and Tidal Energy Conference (EWTEC), 2011. Southampton, UK.

[8] T. Stallard, R. Collings, T. Feng, J. Whelan, Interactions between tidal turbine wakes: experimental study of a group of three-bladed rotors, Philosophical Transactions of the Royal Society A: Mathematical, Physical and Engineering Sciences 371 (2013).

[9] R. Malki, I. Masters, A. Williams, T. Croft, The influence on tidal stream turbine spacing on performance, in: 9th European Wave and Tidal Energy Conference (EWTEC), 2011. Southampton, UK.

[10] D. O'Doherty, A. Mason-Jones, C. Morris, T. O'Doherty, C. Byrne, P. Prickett, R. Grosvenor, Interactions of marine turbines in close proximity, in: 9th European Wave and Tidal Energy Conference (EWTEC), 2011. Southampton, UK.

[11] M. J. Churchfield, Y. Li, P. J. Moriarty, A large-eddy simulation study of wake propagation and power production in an array of tidal-current turbines, Philosophical Transactions of the Royal Society A: Mathematical, Physical and Engineering Sciences 371 (2013). 
[12] T. Divett, R. Vennell, C. Stevens, Optimization of multiple turbine arrays in a channel with tidally reversing flow by numerical modelling with adaptive mesh, Philosophical Transactions of the Royal Society A: Mathematical,Physical and Engineering Sciences 371 (February 28, 2013).

[13] M. Harrison, W. Batten, L. Myers, A. Bahaj, Comparison between CFD simulations and experiments for predicting the far wake of horizontal axis tidal turbines, IET Renewable Power Generation 4 (2010) 613-627.

[14] G. Trowse, R. Krasten, Bay of fundy tidal energy development - opportunities and challenges, Proceeding of the 3rd International Conference on Ocean Energy, Bilbao, Spain, October 6-8 (2010).

[15] R. Karsten, A. Swan, J. Culina, Assessment of arrays of in-stream tidal turbines in the bay of fundy, Philosophical Transactions of the Royal Society A: Mathematical,Physical and Engineering Sciences 371 (February 28, 2013).

[16] T. Roc, D. C. Conley, D. Greaves, Methodology for tidal turbine representation in ocean circulation model, Renewable Energy 51 (2013) 448 - 464.

[17] G. Pinon, P. Mycek, G. Germain, E. Rivoalen, Numerical simulation of the wake of marine current turbines with a particle method, Renewable Energy 46 (2012) 111 - 126.

[18] P. Mycek, B. Gaurier, G. Germain, G. Pinon, E. Rivoalen, Numerical and experimental study of the interaction between two marine current turbines, International Journal of Marine Energy 1 (2013). Accepted manuscript.

[19] G. Germain, A. S. Bahaj, C. Huxley-reynard, P. Roberts, Facilities for marine current energy converter characterization, in: 7th European Wave and Tidal energy Conference, 2007. URL: http://eprints.soton.ac.uk/75855/. 